\title{
Isospin Dependence of Power Corrections in Deep Inelastic Scattering.
}

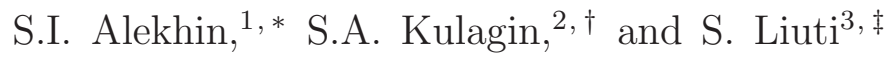 \\ ${ }^{1}$ Institute of High Energy Physics, Protvino, Russia \\ ${ }^{2}$ Institute for Nuclear Research, 117312 Moscow, Russia \\ ${ }^{3}$ University of Virginia, Charlottesville, Virginia 22901, USA.
}

\begin{abstract}
We present results of a perturbative QCD analysis of deep inelastic measurements of both the deuteron and proton structure functions. We evaluate the theoretical uncertainty associated to nuclear effects in the deuteron, and we extract simultaneously the isospin depedendence of: $i$ ) the higher twists terms; ii) the ratio of the longitudinal to transverse cross sections, $R=\sigma_{L} / \sigma_{T}$; iii) the ratio of the neutron to proton structure functions, $F_{2}^{n} / F_{2}^{p}$. The extraction of the latter, in particular, has been at the center of an intense debate. Its accurate determination is crucial both theoretically and for the interpretation of the more precise neutrino experiments including the newly planned high intensity $50 \mathrm{GeV}$ proton synchrotron.
\end{abstract}

PACS numbers: 13.60.Hb, 12.38.Qk

*alekhin@sirius.ihep.su

kulagin@ms2.inr.ac.ru

†sl4y@virginia.edu 


\section{INTRODUCTION}

Deep Inelastic Scattering (DIS) experiments provide the most accurate measurements of the strong coupling constant, $\alpha_{S}$, at intermediate scales. They are also the main source of information on the Parton Distribution Functions (PDFs) in the proton and neutron. The precision with which both of these quantities are known reflects directly into the precision of calculations of the cross sections for all other hard scattering processes. An accurate determination of these, in turn, plays a key role both in the extraction of possible contributions of new physics at new collider energies, and in the interpretation of the forthcoming high precision experiments using neutrino beams [1].

Both $\alpha_{s}$ and PDFs are not directly observable and they need to be extracted from the DIS data according to some procedure. A number of uncertainties affect the analysis, related to both the perturbative QCD (pQCD) series - inclusion of higher orders, threshold resummation effects - and to corrections that are non-perturbative in nature - Target Mass Corrections (TMC), dynamical power corrections, and nuclear effects in the case of the neutron structure function.

It is therefore mandatory to be able to control the size of these uncertainties by introducing a systematic, well tested, method of extraction in which possible ambiguities can be properly gauged. While analyses along these lines exist for the proton structure function (2] in the DIS region and [3] in the "few GeV" or resonance region), an accurate and complete treatment of the neutron structure function is still lacking. This paper is devoted to the application of a newly developed method to determine the isospin dependence of the nucleon Structure Functions (SFs). With the analysis presented here we hope to contribute to the interpretation of both recent data and new experiments, by providing a quantitative measure of the space in which pQCD based corrections and nuclear effects can be wiggled.

For our analysis we use the extensive DIS measurements that exist for both proton and deuteron targets in a wide range of kinematics with the exception for the very large $x$ region where fewer data sets exist, mostly at low values of the final state invariant mass, $W^{2}$, in the region of nucleon resonances (more experiments are however being planned that will cover the large $x$ DIS region in forthcoming programs at Jefferson Lab [4], and at neutrino facilities [1]).

In QCD, different contributions to the DIS structure functions can be written using the Operator Product Expansion (OPE), by ordering them according to their twist, $\tau(\tau=$ dimension - spin) [5]. The Leading Twist (LT) contribution (with $\tau=2$ in DIS) is directly related to the single particle properties of quarks and gluons inside the nucleon - the PDFs. The Higher Twist (HT) components $(\tau=4,6, \ldots$ in unpolarized DIS) involve interactions between quarks and gluons in the nucleon and they are suppressed by terms of order $1 / Q^{2}, 1 / Q^{4} \ldots$, respectively. In phenomenological studies, the PDFs are extracted from QCD global fits. Accurate extractions use data with sufficiently high $Q^{2}$ and invariant mass $W^{2}$, where both target mass and HT corrections are expected to be very small. QCD fits can now be performed to order $\alpha_{S}^{3}$ (Next-to-Next-to-Leading Order, NNLO approximation). If the data encompass a large range in $Q^{2}$, higher order corrections as well as HT effects need to be taken into account simultaneously.

In a recent series of papers [2] a proper choice of the statistical estimator allows one to propagate all experimental error into the uncertainties in the PDFs. Because of the statistical efficiency of this new estimator, the overall systematic error on the PDFs is sensibly reduced with respect to previous analyses based on simplified estimators. With a better de- 
termination of systematic experimental errors in hand, one can address in detail the sources of theoretical errors. Theoretical uncertainties are in principle an elusive concept as by definition they refer to quantities that have yet to be calculated. Uncertainties/ambiguities of this type and inherent to the pQCD analyses are due to: $i$ ) impact of the higher order QCD corrections; ii) the HT terms; iii) target mass corrections (TMC); iv) heavy quarks masses and threshold values; $v$ ) the form of the initial PDFs. These questions were addressed in detail in the analysis of [2], where the DIS cross sections were fitted by including both the LT terms calculated from the PDFs evolved to the NNLO QCD, and the twist-4 terms, evaluated separately for the proton and neutron structure functions $F_{2}$ and $F_{L}$. The fact that the cross sections data were fitted instead of the data on $F_{2}$, allowed for a better determination of $F_{L}$, which in 2] was obtained iteratively. In summary, the analysis of [2] shows that theoretical uncertainties from the pQCD series are under control, and that, due to the new estimator, all extracted quantities can be determined with smaller errors than in previous analyses.

An additional uncertainty is however present in analyses of the isospin dependence of both PDFs and the HT terms, in that the neutron structure functions have to be extracted from nuclear data. The main thrust of our analysis has been to make a thorough assessment of the impact of nuclear corrections on both the LT and HT terms. Here, in particular, we focus on the isospin dependence of the HT terms. Detailed results on both the ratio $F_{2}^{n} / F_{2}^{p}$ and on $R=\sigma_{L} / \sigma_{T}$ will be presented in a forthcoming paper [6]. In our analysis we use the deuteron data where uncertainties are expected to be in better control. We address uncertainties arising from: i) Different models of nuclear effects - we highlight in particular the differences with using the extrapolation [7] of the nuclear density model of the EMC effect [8]; ii) Different deuteron wave functions derived from currently available NN potentials, giving rise to different amounts of high momentum components; iii) The interplay between nucleon off-shellness and TMC in nuclei.

In addition to its practical purpose, a quantitative determination of the isospin dependence of the HTs contributions is of theoretical interest in understanding the nature of power corrections. On one side, infrared renormalons (IRR) have been suggested as a method for estimating the contribution of power corrections to the cross sections for a number of hard processes (see [9] and references therein). Based on this hypothesis the calculations in [10, 11] have predicted the $x$ dependence of the coefficients of the HT terms for both $F_{L}$ and the valence and singlet contributions to $F_{2}$. On the other hand, some models exist that predict a sizeable isospin dependence of the HT terms. This has been suggested for instance in models that interpolate between partonic and non-partonic degrees of freedom at low $Q^{2}$ as in [12] (a smaller effect seems to occur, however, in the predictions for the HT isospin dependence in [13]). A large effect of about a factor two for the ratio of the neutron to proton HT terms, was also predicted based on quark counting estimates in Ref. 14].

A thorough analysis of the isospin dependence of HTs might therefore help disentangling predictions of different models, by investigating, for instance, whether the onset of "flavorblind" power corrections from IRR occurs at a different scale than for other dynamical ones.

In what follows, we present our results for each quantity along with a discussion of the nature of the nuclear effects and the extraction method. In Section [we present the general formalism and definitions. In Section III we discuss the contribution of nuclear effects. In Section IV we outline the extraction method and we present our results. In Section $\nabla$ we discuss some phenomenological applications of our analysis. Finally, we draw our conclusions 
in Section VI.

\section{DIS FORMALISM}

The inclusive DIS section of unpolarized charged leptons off an unpolarized nucleon or nuclear target is fully determined by the spin-averaged electromagnetic tensor of the target, which can be parameterized in terms of two invariant structure functions $F_{1}$ and $F_{2}$ (we ignore a small contribution due to neutral current)

$$
\begin{aligned}
W_{\mu \nu}(p, q) & =\frac{1}{8 \pi} \sum_{\mathrm{s}} \int \mathrm{d}^{4} z \exp (i q \cdot z)\left\langle p, s\left|\left[J_{\mu}^{\mathrm{em}}(z), J_{\nu}^{\mathrm{em}}(0)\right]\right| p, s\right\rangle \\
& =-\widetilde{g}_{\mu \nu} F_{1}+\frac{\widetilde{p}_{\mu} \widetilde{p}_{\nu}}{p \cdot q} F_{2},
\end{aligned}
$$

where $J_{\mu}^{\mathrm{em}}$ is electromagnetic current, $p$ and $q$ are the target momentum and four-momentum transfer, respectively. In order to simplify notations, we denote

$$
\begin{aligned}
\widetilde{g}_{\mu \nu} & =g_{\mu \nu}-\frac{q_{\mu} q_{\nu}}{q^{2}} \\
\widetilde{p}_{\mu} & =p_{\mu}-\frac{p \cdot q}{q^{2}} q_{\mu} .
\end{aligned}
$$

The normalization of states adopted here is $\left\langle p \mid p^{\prime}\right\rangle=2 p_{0}(2 \pi)^{3} \delta\left(\boldsymbol{p}-\boldsymbol{p}^{\prime}\right)$ for both the bosons and fermions. With this normalization the structure functions $F_{1,2}$ are dimensionless. They depend on two invariant variables, namely the Bjorken variable $x=Q^{2} / 2 p \cdot q$ and the four-momentum transfer squared $Q^{2}=-q^{2}$.

The differential cross section in terms of the structure functions and standard variables $x$ and $y=p \cdot q / p \cdot k$, where $k$ is the incoming lepton four-momentum, reads

$$
\frac{\mathrm{d}^{2} \sigma}{\mathrm{d} x \mathrm{~d} y}=\frac{4 \pi \alpha^{2}}{Q^{2} x y}\left[\left(1-y-\frac{(M x y)^{2}}{Q^{2}}\right) F_{2}+\frac{1}{2} y^{2}\left(1-\frac{2 m_{l}^{2}}{Q^{2}}\right) F_{T}\right],
$$

where $\alpha$ is the electromagnetic coupling constant, $M$ is the nucleon mass and $Q^{2}=2 x y p \cdot k$, and $F_{T}=2 x F_{1}$. [50].

In QCD the contributions from different quark-gluon operators to the electromagnetic tensor and to DIS structure functions are ordered according to their twist, leading to the expansion in inverse powers of $Q^{2}$ :

$$
F_{T, 2}\left(x, Q^{2}\right)=F_{T, 2}^{L T}\left(x, Q^{2}\right)+\frac{H_{T, 2}\left(x, Q^{2}\right)}{Q^{2}}+\mathcal{O}\left(1 / Q^{4}\right) .
$$

This expansion applies to both the proton and neutron structure functions, we have suppressed the indices for simplicity. The first term is the LT contribution and $H_{1,2}$ are the HT - twist-4 - contributions. Furthermore, if a finite mass for the nucleon target is considered, new terms arise in Eq.(5) that mix operators of different spin, leading to additional power terms of kinematical origin - the so-called target mass corrections. In the approximation that $x^{2} M^{2} / Q^{2}$ is small, the TMC series can be absorbed in the leading twist term [15].

The separation of LT, TMC and dynamical HT from the data, is not straightforward as witnessed by the number of studies dedicated to it since the initial formulation of the problem in the 1970's [15, 16]. In what follows we define the LT, TMC, and HT, contributions to the structure functions $F_{T}$ and $F_{2}$. The formalism for nuclear DIS is discussed in Section ஹI 


\section{A. Leading Twist}

The LT part of the structure functions is related to the PDFs, $p_{i}(x, Q)$ - the index $i$ refers to the different types of quarks and antiquarks, and to the gluon distribution - via a convolution with perturbatively calculable coefficient functions $C_{T, 2}^{i}$ :

$$
F_{T, 2}^{\mathrm{LT}}\left(x, Q^{2}\right)=\sum_{i=q, \bar{q}, g} \int_{x}^{1} \frac{\mathrm{d} z}{z} C_{T, 2}^{i}\left(z, \alpha_{S}\left(Q^{2}\right)\right) p_{i}\left(x / z, Q^{2}\right) .
$$

The $Q^{2}$ dependence of the PDFs is predicted by the well-known DGLAP evolution equations [17]:

$$
t \frac{\partial p_{i}(x, t)}{\partial t}=\sum_{j=q, \bar{q}, g} \int_{x}^{1} \frac{\mathrm{d} z}{z} P_{i j}\left(z, \alpha_{S}(t)\right) p_{j}(x / z, t),
$$

where $t=Q^{2}$, and $\alpha_{S}$ is the strong coupling constant, and $P_{i j}$ are the splitting functions.

The coefficient functions have been calculated to NNLO [18]. The splitting functions are known to NLO, and only a limited set of Mellin moments are evaluated to NNLO [20]. Although, estimates of the full $x$-dependence of the splitting functions in NNLO approximation are available [19], in our analysis we use the $\overline{\mathrm{MS}}$ NLO QCD approximation with the renormalization/factorization scales chosen equal to $Q$. The NNLO variant of our fit is used to estimate the uncertainty due to higher orders. Large $x$ resummation effects, arising from terms of the type $\left[\alpha_{S}\left(Q^{2}\right) \ln (1-z)\right]^{2 k}$ at $\mathcal{O}\left(\alpha_{s}^{k}\right)$, in the coefficient functions are present in principle. They have been shown to be comparable in size to NNLO corrections in Ref. [21], and to generate a further negative correction to the HT coefficient of the proton SF.

\section{B. Target Mass Corrections}

We follow the method of Ref. 15] where it was shown that the TMC series can be summed up, leading to the modification of the LT term. Therefore, Eq. (5.5) remains valid with the LT terms replaced by

$$
\begin{aligned}
& F_{T}^{\mathrm{LT}, \mathrm{TMC}}\left(x, Q^{2}\right)=\frac{x^{2}}{\xi^{2} \gamma} F_{T}^{\mathrm{LT}}\left(\xi, Q^{2}\right)+2 \frac{x^{3} M^{2}}{Q^{2} \gamma^{2}} \int_{\xi}^{1} \frac{\mathrm{d} \xi^{\prime}}{\xi^{\prime 2}} F_{2}^{\mathrm{LT}}\left(\xi^{\prime}, Q^{2}\right), \\
& F_{2}^{\mathrm{LT}, \mathrm{TMC}}\left(x, Q^{2}\right)=\frac{x^{2}}{\xi^{2} \gamma^{3}} F_{2}^{\mathrm{LT}}\left(\xi, Q^{2}\right)+6 \frac{x^{3} M^{2}}{Q^{2} \gamma^{4}} \int_{\xi}^{1} \frac{\mathrm{d} \xi^{\prime}}{\xi^{\prime 2}} F_{2}^{\mathrm{LT}}\left(\xi^{\prime}, Q^{2}\right),
\end{aligned}
$$

where $\gamma=\left(1+4 x^{2} M^{2} / Q^{2}\right)^{1 / 2}$ and $\xi=2 x /(1+\gamma)$ is the Nachtmann variable [22].

It must be noted however, that the derivation of [15] was given in the zeroth order in $\alpha_{S}$, assuming that the target quarks are on-shell. Both, higher order $\alpha_{S}$ corrections and quark off-shell effects modify Eqs.(8). It was argued in [23] that off-shell effects lead to $M^{2} / Q^{2}$ terms which are not incorporated in Eqs.(8). In addition, target mass corrections should be applied also to the HT terms in the higher order terms in the expansion (5). For this reason we do not consider $1 / Q^{4}$ terms in the TMC formula, which are small for the kinematical range considered. Finally, TMC corrections for an off-shell target, i.e. when $p^{2} \neq M^{2}$, should be treated as part of the nuclear effects and will be discussed therefore in Section III. 


\section{Higher Twists}

The extraction of higher twist terms from the data is a longstanding problem, as recognized from the very first developments of a pQCD phenomenology $([16,24])$. HTs have been hard to pin down for a number of reasons. First of all a connection with partonic interpretations cannot be established on a one by one basis, differently from the LT components that are directly related to the PDFs. In fact the HT terms are formally written within OPE as the product of coefficient functions and hadronic matrix elements of composite local operators. Not all of the matrix elements are independent, but a minimal basis can be selected after relating them through the equations of motion [25]. Nevertheless, even in a minimal basis, the number of independent reduced matrix elements is much larger than the number of observables - e.g. the moments of the structure functions. In unpolarized scattering one can single out formally the four quarks and two quarks-two gluons types of operators, corresponding in a partonic language to quark-quark and quark-gluon correlations, respectively. The determination of the relative scales of these contributions is at present model dependent. However, it was shown in Ref. [26] that a simultaneous analysis of both $F_{2}$ and $F_{L}$ better constrained the evaluation of the quark-gluon term, that was required to be large and negative in order to fit both sets of data.

A practical difficulty is also in that theoretical estimates - from the simple estimate of the increase of the number of operators with respect to twist-2 [24], to more sophisticated IRR calculations [10, 11] - predict that HTs are most important at low $W^{2} \approx Q^{2}(1-x)$. In this limit, with $W^{2} \geq 4 \mathrm{GeV}^{2}$, thus avoiding the resonance region, it can be easily shown that the logarithmic dependencies characterizing pQCD evolution to a given order mimic the $1 / Q^{2}$ dependence of the twist- 4 terms. A correlation between the pQCD parameters and the HT coefficients arises that has lead the authors of [27] to conclude that for the structure function $F_{3}$, the NNLO term and the HT corrections are, within the precision of current data, undistinguishable. A similar analysis was subsequently applied in Ref.[7] to $F_{2}$. It lead the authors to the conclusion that HTs are highly reduced with respect to previous determinations, even inside the resonance region.

To summarize, the results of a number of analyses of the HT contributions in DIS are still not conclusive. A joint search using a combination of predictions from hadronic models and an accurate experimental extraction seems to be the most promising avenue. It is in this spirit that in this paper we address yet another aspect of the phenomenology of HTs, namely their isospin dependence. An isospin dependence of the HT terms resides entirely in their non-perturbative structure. In IRR models in fact the coefficients of the HT terms are predicted to have no target dependence, provided the radiative corrections to these terms are factored out by assuming for instance, that they behave similarly to their LT correspondent. An assessment of the magnitude of the isospin dependence of the HT terms provides therefore a handle on understanding their nature and more precisely the extent to which they can be described by models. In particular, possible scenarios about the large $x$ structure of the proton can be investigated, envisaging coherent scattering from multiquark composites carrying increasing momentum fraction at $x \rightarrow 1$ [14].

\section{NUCLEAR EFFECTS}

Since nuclear data are used in the QCD analysis, nuclear effects must be taken into account. In our analysis we use deuterium data in order to study the isospin effect in PDFs 
and HT terms. In this section we briefly discuss our method to correct for nuclear effects. More details can be found elsewhere [6].

\section{A. Fermi motion and binding effects}

For large $x>0.1$, away from nuclear shadowing region, nuclear DIS of leptons off nuclear targets can be viewed as incoherent scattering off bound nucleons [28, 29, 30, 31, 32, 33]. The DIS cross section is given by the imaginary part of the virtual photon Compton amplitude in the forward direction. In incoherent scattering approximation, the nuclear Compton amplitude is taken in impulse approximation by disregarding both initial state interactions and interactions between the struck quark and the nuclear debris. The corresponding Feynman diagram is shown in Fig 1 Calculating this diagram and projecting the structure functions from the imaginary part of the Compton amplitude we derive the relations between the nuclear and the nucleon structure functions. For the deuteron we have

$$
\begin{aligned}
x F_{T}^{D}\left(x, Q^{2}\right)= & \int \frac{\mathrm{d}^{3} \boldsymbol{p}}{(2 \pi)^{3}}\left|\Psi_{D}(\boldsymbol{p})\right|^{2}\left(1+\frac{\gamma p_{z}}{M}\right) \\
& \left(x^{\prime} F_{T}^{N}\left(x^{\prime}, Q^{2} ; p^{2}\right)+\frac{x^{\prime 2} \boldsymbol{p}_{\perp}^{2}}{Q^{2}} F_{2}^{N}\left(x^{\prime}, Q^{2} ; p^{2}\right)\right), \\
\gamma^{2} F_{2}^{D}\left(x, Q^{2}\right)= & \int \frac{\mathrm{d}^{3} \boldsymbol{p}}{(2 \pi)^{3}}\left|\Psi_{D}(\boldsymbol{p})\right|^{2}\left(1+\frac{\gamma p_{z}}{M}\right) \\
& \left(1+\frac{4 x^{\prime 2}\left(p^{2}+\frac{3}{2} \boldsymbol{p}_{\perp}^{2}\right)}{Q^{2}}\right) F_{2}^{N}\left(x^{\prime}, Q^{2} ; p^{2}\right),
\end{aligned}
$$

where $F_{2, T}^{N}=\left(F_{2, T}^{p}+F_{2, T}^{n}\right) / 2$. The deuteron wave function $\Psi_{D}(\boldsymbol{p})$ squared describes the probability to find the bound proton (or neutron) with momentum $\boldsymbol{p}, x^{\prime}=Q^{2} / 2 p \cdot q$ is the Bjorken variable of the bound nucleon with the four-momentum $p$, which is given by the difference of the target four-momentum and the four-momentum of the spectator nucleon. Eqs.(9) are written for the target rest frame and the $z$ axis is chosen such that the momentum transfer, $q=\left(q_{0}, \mathbf{0}_{\perp},-|\boldsymbol{q}|\right)$. In this reference frame $p=\left(M_{D}-\sqrt{\boldsymbol{p}^{2}+M^{2}}, \boldsymbol{p}_{\perp}, p_{z}\right)$, where $M_{D}$ and $M$ are the deuteron and the nucleon mass respectively. The kinematical factors in Eqs. (9) result from the projection of the structure functions from the hadronic tensor. The transverse motion of the bound nucleon in the deuteron rest frame is the reason for the appearance of additional terms in the transverse and the longitudinal cross sections, i.e. the terms proportional to $x^{\prime 2} \boldsymbol{p}_{\perp}^{2} F_{2}$ in Eqs.(9).

\section{B. Off-shell effects}

The bound proton and neutron are off-mass-shell and their structure functions differ from those of the free proton and neutron. The off-shell nucleon structure functions depend on the nucleon virtuality $p^{2}$ as an additional variable. Therefore, off-shell effects in the structure functions are closely related to the target mass corrections. Target mass effects in the off-shell nucleon can be of two different kinds. First, similarly to the on-shell nucleon, we have to take into account the kinematical target mass dependence due to the finite $p^{2} / Q^{2}$

ratio. We assume that this effect is described by Eqs. (8), where the nucleon mass squared is 
replaced by $p^{2}$ (this leads in turn to the modification of the parameter $\gamma$ and the variable $\xi$ in the off-shell region). Furthermore, the dependence on $p^{2}$ appears already at leading twist 32, 33, 34, 35]. In order to estimate the off-shell effect in the LT structure functions, we consider the quark distribution in terms of the spectral representation [32, 33, 34]

$$
\begin{aligned}
q\left(x, p^{2}\right) & =\int \mathrm{d} s \int \mathrm{d} k^{2} \rho\left(s, k^{2}, x ; p^{2}\right), \\
k_{\max }^{2}(x, s) & =x\left(p^{2}-\frac{s}{1-x}\right) .
\end{aligned}
$$

The integration in Eq.(10) is taken over the mass spectrum of spectator states $s$ and quark virtuality $k^{2}, k_{\max }^{2}$ is the kinematical maximum of $k^{2}$ for the given $s$ and $p^{2}$. The invariant spectral density $\rho$ measures the probability to find in a nucleon with momentum $p$, a quark with light-cone momentum $x$ and virtuality $k^{2}$ and the remnant system in a state with invariant mass $s$.

We observe from Eq.(10) that the $p^{2}$ dependence of quark distributions has two primary sources: the one in the upper limit of $k^{2}$ integration (kinematical off-shell dependence), and an explicit $p^{2}$ dependence of the quark spectral function $\rho$ (dynamical off-shell dependence).

The kinematical off-shell effect causes a negative correction to the bound nucleon structure functions and produces an enhanced EMC effect, as first noticed in 32, 33]. However, if only the kinematical off-shell effects are taken into account the number of valence quarks in the nucleon would change with $p^{2}$. It can be seen directly from Eq.(10) that the normalization of the quark distribution decreases as $p^{2}$ decreases, provided that the spectral density is positively defined. This effect leads to an overall $1-2 \%$ depletion of valence quarks in the deuteron. Furthermore, the magnitude of this effect increases in heavy nuclei, since the average shift from the mass shell of the bound nucleon increases. This observation indicates that an off-shell effect of dynamical origin must also be present.

Dynamical off-shell effects can be viewed as a measure of the nucleon's deformation inside the nuclear medium. One possible way to evaluate dynamical off-shell effects is to require the conservation of the valence quark number in the nucleon also in the off-shell region [33, 34]:

$$
\frac{\mathrm{d}}{\mathrm{d} p^{2}} \int_{0}^{1} \mathrm{~d} x q_{\mathrm{val}}\left(x ; p^{2}\right)=0,
$$

This equation makes it possible to estimate the off-shell effect minimizing the model dependence [33]. It was shown that Eq.(12) results in a partial cancellation between kinematical and dynamical off-shell effects [33, 34]. However, the off-shell effect in the structure functions remains an important correction.

\section{Results and comparison with other approaches}

The effect of nuclear corrections is illustrated in Fig 2 The ratio $R_{2}^{D}\left(x, Q^{2}\right)=$ $F_{2}^{D}\left(x, Q^{2}\right) / F_{2}^{N}\left(x, Q^{2}\right)$ was calculated by Eqs.(9) using different approximations. The dotted curve corresponds to the standard assumption that the bound proton and neutron structure functions in Eqs.(9) are equal to those of the free nucleon ones. The competition between nuclear binding and Fermi motion determines the shape of $R_{2}$. In particular, the depletion of nuclear structure functions at $x<0.7$ is due to the effect of nuclear binding, while the 
rise of the $R_{2}$ ratio at large $x$ is due to the effect of the nucleon momentum distribution (Fermi motion) [28].

The dashed curve corresponds to the results with the target mass corrections. We notice that TMC is an important correction at large $x$, as can be directly seen from Eqs. (8). This correction modifies the shape and the magnitude of the LT structure function at large $x$. This in turn leads to the softening of the ratio $R_{2}^{D}$ at large $x$.

The solid curve stands for the full calculation with TMC and off-shell effect taken into account. We observe, that the off-shell effect is most important in the binding region $(x$ between 0.3 and 0.7 ), where it causes a negative correction to the bound nucleon structure functions.

The region of large $x$ corresponds to small masses of produced hadronic states $W^{2}=$ $M^{2}+Q^{2}(1 / x-1)$. For instance, the events with $Q^{2}=10 \mathrm{GeV}^{2}$ and $x>0.75$ fall into the resonance region. For this reason the DIS parametrization of structure functions, which are used in computing the nuclear effects with Eq.(9), are questionable at large $x$. In order to avoid the resonance region and elucidate nuclear effects in the DIS regime, we apply a cut at $W=1.8 \mathrm{GeV}$. The ratio $R_{2}^{D}$ as a function of $W$ was calculated for a few different values of $Q^{2}$. The results are shown in the lower panel of Fig 2

The off-shell effect is much less important in the deuteron than in heavy nuclei. The strength of this effect is governed by the average off-shellness of the bound nucleon $\Delta=$ $\left\langle p^{2}-M^{2}\right\rangle / M^{2} \approx 2(\varepsilon-T) / M$, where $T=\left\langle\boldsymbol{p}^{2}\right\rangle / 2 M$ and $\varepsilon=\left\langle p_{0}\right\rangle-M$ are the average kinetic and separation energy. In order to illustrate the strength of nuclear binding and off-shell effects, we have calculated $T, \varepsilon$, and $\Delta$ averaged over the nuclear spectral function for a number of nuclei. In Fig 3, these parameters are plotted as a function of the nuclear masss number $A$. It can be seen that $\Delta$ increases by a factor of five when going from the deuteron to heavy nuclei.

A phenomenological model of the EMC effect in the deuteron was given in Ref. [36]. The model is based on an extrapolation of SLAC data on the EMC effect for a number of nuclei from ${ }^{4} \mathrm{He}$ to ${ }^{197} \mathrm{Au}$, where the key assumption was made that the quantity $\left(F_{2}^{A} / F_{2}^{N}-1\right)-$ $F_{2}^{A}$ being the nuclear structure function per nucleon - scales with the nuclear density:

$$
\frac{F_{2}^{D} / F_{2}^{N}-1}{F_{2}^{A} / F_{2}^{N}-1}=\frac{\rho_{d}}{\rho_{A}}
$$

where $\rho_{d}$ and $\rho_{A}$ are the number densities in the deuteron and in a heavy nucleus (Nuclear Density Model (NDM) 8] ). It was also assumed that this ratio is independent of $Q^{2}$. Using Eq.(13), the ratio $F_{2}^{D} / F_{2}^{N}$ was obtained in Ref.[36] in terms of the experimentally measured ratio $F_{2}^{A} / F_{2}^{D}$ by using:

$$
\frac{F_{2}^{D}}{F_{2}^{N}} \approx 1+\frac{\rho_{d}}{\rho_{A}-\rho_{d}}\left(\frac{F_{2}^{A}}{F_{2}^{D}}-1\right)
$$

and by averaging the quantity appearing on the r.h.s. over the SLAC nuclear data. The values of $F_{2}^{D} / F_{2}^{N}$ were given for $x$ corresponding to the data bins. These results are shown in Fig 2 by a shaded region.

We observe that the NDM attempts to extrapolate "density scaling" to the region of light nuclei, where the notion of density is ill defined [37]. The value $\rho_{d}=0.024 \mathrm{fm}^{-3}$ was used in Ref. [36], which was derived using the r.m.s. radius of the deuteron. However, it is not clear what volume is occupied by the deuteron and for this reason $\rho_{d}$ has a large "theoretical" 
uncertainty. Since the quantity $F_{2}^{D} / F_{2}^{N}-1$ is proportional to $\rho_{d}$, this theoretical uncertainty will directly translate into an uncertainty for the extrapolated ratio $F_{2}^{D} / F_{2}^{N}$. This was not given in Ref. [36] and it is likely to be larger than the errors shown in Fig 2

\section{QCD ANALYSIS AND FIT}

\section{A. Fitting Procedure}

In our analysis the data on charged lepton DIS off proton and deuterium targets from BCDMS, NMC, H1, ZEUS, and SLAC experiments were used [38]. The cut $Q^{2}>2.5 \mathrm{GeV}^{2}$ was applied in order to avoid the region where $\alpha_{s}$ is large and the higher-order QCD radiative corrections can be out of control. The HERA data with $Q^{2}>250 \mathrm{GeV}^{2}$ were not used in the analysis since the impact of those data on the fit is marginal because of large experimental errors. The maximum $x$ in the data set is about 0.9 and minimum center-of-mass energy $W$ is about $1.8 \mathrm{GeV}$ (see Fig (4). The total number of data points (NDP) is 1381 for the proton and 998 for the deuteron; $\chi^{2} / N D P=1.1$ for the best fit.

In our fit we make use of Eq.(4) with the LT structure functions given in Eq. (6) corrected for the target mass effect by Eq. (8). The higher twist terms $H_{2, T}$ of Eq.(15), the parton distributions, and the value of $\alpha_{\mathrm{s}}$ were simultaneously fitted to the data. The parton distributions were parametrized in the form used in the earlier analyses of Ref. [2] with initial scale of the QCD evolution $Q_{0}^{2}=9 \mathrm{GeV}^{2}$. The evolution equations were solved numerically by direct integration in $x$-space. Our procedure is in agreement with the benchmarks introduced in Ref. [39] that require the precision of the solution to be much better than the accuracy of the data used in the analysis.

The implementation of target mass and nuclear corrections in our fit is as follows. The proton and neutron structure functions were calculated as

$$
F_{2, \mathrm{~T}}^{p, n}=\mathcal{T}_{2, T}\left\{F_{2, \mathrm{~T}}^{p, n(\mathrm{LT})}\right\}+H_{2, \mathrm{~T}}^{p, n} / Q^{2},
$$

where we have expressed the target mass correction formula given in Eq.(8) in terms of the functional: $\mathcal{T}_{2, T}$.

The deuteron structure functions were calculated as

$$
F_{2, \mathrm{~T}}^{D}=\mathcal{F}_{2, T}\left\{F_{2, \mathrm{~T}}^{p}+F_{2, \mathrm{~T}}^{n}\right\},
$$

where $\mathcal{F}_{2, T}$ are the nuclear smearing functionals corresponding to Eq.(9), and $F_{2, T}^{p, n}$ are the proton and neutron structure functions of Eq.(15). However, the implementation of this approach slows down the numerical calculation because of 4-dimensional integrations in the deuteron cross sections in terms of the QCD-evolved PDFs. We are able to reduce the calculation time by using approximate expressions for the deuteron structure functions:

$$
\begin{aligned}
F_{2, \mathrm{~T}}^{D}= & \frac{\mathcal{F}_{2, \mathrm{~T}}\left\{F_{2, \mathrm{~T}}^{p(Q P M)}+F_{2, \mathrm{~T}}^{n(Q P M)}\right\}}{F_{2, \mathrm{~T}}^{p(Q P M)}+F_{2, \mathrm{~T}}^{n(Q P M)}} \times \\
& {\left[\frac{\mathcal{T}_{2, \mathrm{~T}}\left\{F_{2, \mathrm{~T}}^{p(Q P M)}+F_{2, \mathrm{~T}}^{n(Q P M)}\right\}}{F_{2, \mathrm{~T}}^{p(Q P M)}+F_{2, \mathrm{~T}}^{n(Q P M)}}\left(F_{2, \mathrm{~T}}^{p(L T)}+F_{2, \mathrm{~T}}^{n(L T)}\right)+\frac{H_{2, \mathrm{~T}}^{p, n}}{Q^{2}}\right], }
\end{aligned}
$$


where $F_{2, \mathrm{~T}}^{\mathrm{QPM}}$ is given by Eq. (15) with the LT term in the "quark-parton model", that is without QCD radiative corrections to the coefficient functions. The deuteron structure functions calculated using Eq.(17) require 3-dimensional integration only. This approximation introduces only a marginal bias in the final results.

We parametrize the functions $H_{2, \mathrm{~T}}(x)$ at the selected values of $x$ and interpolate between those grid points using cubic splines. The positions of the grid points were selected in such a way to provide the overlap between the error-bars of nearest grid points. The values of the functions $H_{2, \mathrm{~T}}(x)$ at the grid points have been fitted to data. This method made it possible to describe the different structure functions in our fit and, at the same time, to keep the number of fitting parameters reasonable.

All experimental errors in the data including uncorrelated statistical, correlated systematical, and errors in overall normalizations have been taken into account in the analysis using the covariance matrix approach. The error bands throughout the paper are due to linear propagation of the errors into the fitted parameters. In several of the experimental data sets used in our analysis the overall normalization has been fixed by the authors from comparisons to other data.

For this reason we introduce an additional normalization parameter for each target (in some cases also for each energy) in the experiment and fit these parameters simultaneously with the parameters of PDFs and the HT terms. The overall normalization errors for such data subsets are accounted by the error propagation in the corresponding normalization factors through the general Hessian matrix of the fit. Such treatment of the normalization errors allows for a maximal self-consistency of the analysis, incorporating all existing information and minimizing the normalizaton uncertainties in the data. The re-normalized data subsets and their re-normalization factors, derived in the fit, are listed in Table【. The re-normalization factors are generally close to 1 for the deuteron data and somewhat higher for the SLAC proton data.

\section{B. Results}

The isospin asymmetries of the HT terms in $F_{2, \mathrm{~T}}$ obtained from this fit are shown in Figs. [5 to 9 as full lines, which correspond to the $1 \sigma$ bands of the total experimental errors [49]. In order to study the sensitivity of the results to various theoretical assumptions and estimate theoretical errors, we performed a number of fits using different approximations and assumptions.

The central value results of these fits are also given in Figs. 5] to 9. Since the errors in $H_{2, \mathrm{~T}}$ obtained in all variants of the fit are correlated, the statistically significant shifts are those whose central values lie out of the bands given by the solid curves.

For all variants of the fit we put the constraint $H_{2, \mathrm{~T}}(x)=0$ as $x \rightarrow 0$, since the analyzed data are not sensitive to the isospin asymmetry at small $x$. Relaxing this constraint does not improve the quality of the fit and in this case the values of $H_{2, \mathrm{~T}}(0)$ are comparable to zero within errors.

The sensitivity of the isospin asymmetry of the HT terms to the treatment of Fermi Motion and nuclear Binding (FMB) in the deuteron is shown in Fig 5 . One observes that different approaches lead to variations in $H_{2}^{n-p}$ up to several standard deviations at $x \gtrsim 0.75$. The high sensitivity of the fit to details of the treatment of Fermi motion indicates that an accurate account of nuclear smearing in deuterium is crucial for the determination of $H_{2, T}^{n-p}$ at large $x$. 
TMC also strongly affect the extraction of the isospin asymmetry due to their interplay with nuclear corrections at large $x$, as it can be seen from Fig 2 This is because TMC modify the $x$ dependence of the LT structure functions at large $x$. The impact of TMC on the deuteron correction is illustrated in Fig [ in which the fit without TMC in $F_{2, \mathrm{~T}}^{p, n(Q P M)}$ in Eq.(17) is compared to the fit with the full treatment of TMC. We observe a noticeable difference between these two fits in the region of $x \approx 0.75$, especially for $H_{2}^{n-p}$.

We have also studied the sensitivity of our result to the choice of the deuteron wave function in Eq.(9). Calculations of deuteron wave functions predict different amounts of high momentum components depending on the nucleon-nucleon potentials that are used, and on the treatment of relativistic effects. We considered two extreme situations by comparing in Fig 7 results using the Bonn [41] and Paris [42] potentials that predict the smallest high momentum components among modern calculations, and a phenomenological wave function that reproduces y-scaling data and that has therefore a larger amount of high momentum components. Relativistic calculations seem also to have a larger amount of high momentum components [40]. One can see that the functions $H_{2, \mathrm{~T}}^{n-p}$ vary within one standard deviation for the Paris and Bonn wave functions. The phenomenological distribution, however, is more than one standard deviation away at $x \geq 0.75$.

The isospin asymmetry in the HT terms is also affected by off-shell effects in the bound nucleon structure functions discussed in Section III. Since the calculation of off-shell effects is model dependent, these effects are in principle the main source of theoretical uncertainty in our analysis. However, the shift from the mass shell for the bound proton and neutron in the deuteron, measured by $\Delta=\left\langle p^{2}-M^{2}\right\rangle / M^{2}$, is small because of the weak binding in the deuteron (see Fig 3). As a result, the net effect of off-shell corrections is within the $H_{2, \mathrm{~T}}^{n-p}$ error-bars (see Fig $[8$ ) and thus the uncertainty from the modeling of off-shell effect is effectively small for the deuteron. We note, however, that the off-shell effects will be much more important in heavy nuclei, as also shown in Fig 3

In order to examine the impact of different models of nuclear effects in DIS, we repeated the fit with the deuteron correction calculated using the Nuclear Density Model (NDM) discussed in Section [II]. Since the NDM correction is not available for $x>0.8$, we have removed the corresponding data points from the fit. Furthermore, since the NDM does not distinguish among structure functions, we assumed the correction to $F_{\mathrm{T}}$ to be the same as for $F_{2}$. The results are shown in Fig 8 . The term $H_{\mathrm{T}}^{n-p}$ obtained from the NDM fit is different from the corresponding term of the FMB fit, while for $H_{2}^{n-p}$ we observe a good agreement between these two fits. However, since the nuclear density model of the EMC effect in the deuteron is essentially different from the approach discussed in this paper, this agreement appears to be accidental.

Another source of theoretical uncertainty comes from higher order QCD radiative corrections. These corrections decrease as $Q^{2}$ increases. For this reason the radiative corrections can simulate the power-like terms in some kinematical regions of $Q^{2}$. Indeed, it is well known that the magnitude of phenomenological HT terms is strongly correlated with the order of the QCD radiative corrections applied in the analysis. The HT terms drastically decrease when going from LO to NLO in the structure function fit. However, the magnitude of the HT terms does not change much when going from NLO to NNLO, the variation of the HT terms stays within one standard deviation 2, 27]. In the present analysis we also observe only a marginal variation of $H_{2, \mathrm{~T}}^{n-p}$ after the NNLO corrections have been applied (see Fig 9). We do not consider the soft gluon re-summation [21] as well as the log-like dependence of $H_{2, \mathrm{~T}}$ because of anomalous dimensions [44]. The isoscalar part of the HT terms can be 
affected by these effects at large $x \sim 0.9$. However, the isovector combinations $H_{2, \mathrm{~T}}^{n-p}$ seem to be much less sensitive to these effects.

To summarize, the isospin asymmetry is stable with respect to the theoretical uncertainties addressed in this paper. The statistical correlations between the LT and the HT terms in our fit are illustrated in Fig.10. A wide kinematical region of considered DIS data allows to reliably separate the LT and HT terms and, as a result, the corresponding correlation coefficients are less than 0.5.

\section{PHENOMENOLOGY}

It is instructive to compare our results to the earlier extraction of the isotopic effects in the HT terms. The NMC extraction of the HT asymmetry in $F_{2}$ [45] is based on the equation

$$
\frac{F_{2}^{D}}{F_{2}^{p}}-1=\frac{\mathcal{T}_{2}\left\{F_{2}^{n(L T)}\right\}}{\mathcal{T}_{2}\left\{F_{2}^{p(L T)}\right\}}\left(1-\frac{C_{2}^{p}-C_{2}^{n}}{Q^{2}}\right),
$$

in which the combined SLAC-NMC-BCDMS data for the deuteron and proton are used. The relation between the functions $C_{2}^{p, n}$ and the functions $H_{2}^{p, n}$ from our analysis can be written as follows

$$
C_{2}^{p}-C_{2}^{n} \approx \frac{H_{2}^{p}}{\mathcal{T}_{2}\left\{F_{2}^{p(L T)}\right\}}-\frac{H_{2}^{n}}{\mathcal{T}_{2}\left\{F_{2}^{n(L T)}\right\}} .
$$

It must be noted, that the correspondence between these two definitions is somewhat uncertain since the denominators in Eq. (18) depend on $Q^{2}$. However for comparison, in Fig [1] we plot the difference $C_{2}^{p}-C_{2}^{n}$ calculated from our results using Eq.(18) at different $Q^{2}$. Both extractions agree within errors. We note that our errors are systematically smaller because of a larger number of data points used in the analysis. It must be also emphasized that our analysis and the one by NMC are different in a few aspects (no deuteron corrections were applied in the NMC analysis, different treatments of systematic errors were used, etc.). All of these factors could be responsible for the remaining discrepancies.

In Fig 12, the isospin asymmetry in the ratio $R=\sigma_{L} / \sigma_{T}$

$$
R=\gamma^{2} \frac{F_{2}}{F_{T}}-1
$$

extracted from the fit of the SLAC data [46], is compared to our results. A good agreement on $R^{D}-R^{p}$ suggests that our fit is self-consistent, since our analysis includes the data used in Ref. [46]. The value of $R^{D}-R^{p}$ at $x=0.03 \div 0.35$ measured by NMC [47] is also comparable to the calculations based on our fit. In Fig 12 we show the different contributions to $R^{D}-R^{p}$ considered in our analysis. It can be seen that at large $x$ the value of $R^{D}-R^{p}$ is defined mainly by the HT terms.

The isotopic asymmetries $H_{2, \mathrm{~T}}^{n-p}$ determined from our fit can be also compared with the predictions of different theoretical models. A popular model is the infrared renormalon model (IRR) of Refs. 10, 11]. In this model HT terms derive from the resummation of multi-loop diagrams, and their $x$ dependence is obtained as the Mellin convolution of the LT terms with flavor-independent coefficient functions $C^{\mathrm{IRR}}$,

$$
H_{2, \mathrm{~T}}=A_{2}^{\prime} C_{2, \mathrm{~T}}^{I R R} \otimes F_{2}^{Q P M} .
$$


The dimensional normalization factor $A_{2}^{\prime}$ determines the characteristic scale of the HT terms. This scale is not determined in the IRR model and it is adjusted from comparisons with the data. In Fig 13, our results are compared to the IRR model calculation for the non-singlet contribution, with the normalization factor $A_{2}^{\prime}=-0.3 \mathrm{GeV}^{2}$. It is clear that the agreement is poor and it cannot be improved by simply varying the normalization factor of the IRR model. Alternatively, the comparison can be made in terms of the Mellin moments

$$
M_{2, \mathrm{~T}}(N)=\int_{0}^{1} d x x^{N-2} H_{2, \mathrm{~T}}(x) .
$$

The Mellin moments of $H_{2, \mathrm{~T}}^{n-p}$ are given in Table VI. In Fig 14, the ratio of these moments to the moments of $F_{2}^{Q P M}$ is plotted. Also shown are the moments of $C_{2, \mathrm{~T}}^{I R R}$. We only show $N \geq 2$, since the data do not allow us to constrain the behavior of $H_{2, T}^{n-p}$ at small $x$. Again, we observe a disagreement with the IRR model. Parameterizations of DIS structure functions interpolating between the low and high $Q^{2}$ regions are also available [12, 13]. The comparison of our results with these models is not directly feasible, because of the strong $Q^{2}$ dependence introduced in their evaluation of the HT terms, due to effects beyond the OPE.

Having estimated the power corrections to different structure functions, one can make an extrapolation into the resonance region in order to study the phenomenon of duality [48]. Examples of such extrapolations are given in Figs 1516, At values of $Q^{2}$ relevant for the experiments at Jefferson Lab the HT terms contribute moderately to the ratio $F_{2}^{n} / F_{2}^{p}$. For the difference $R^{D}-R^{p}$ the impact of the HT terms is larger. At $W \lesssim 1.4 \mathrm{GeV}$ the dominant contribution comes from $H_{2}^{n-p}$. At $W \gtrsim 1.4 \mathrm{GeV}$ the contribution from $H_{2}^{n-p}$ is small and the main effect comes from $H_{\mathrm{T}}^{n-p}$. However the errors are large in this region, since it corresponds to $x \sim 0.4$, where $H_{\mathrm{T}}^{n-p}$ has its largest uncertainty.

\section{CONCLUSIONS}

In conclusion, we determined the isospin asymmetries of the functions $H_{2}$ and $H_{\mathrm{T}}$ describing the HT terms of the DIS structure functions. The value of $H_{\mathrm{T}}$ is consistent with zero within the errors for all values of $x$. Also $H_{2}$ is consistent with zero at low and intermediate values of $x$; It deviates from zero at $x \gtrsim 0.7$. We performed a careful study of the theoretical uncertainties that might affect the extraction and we conclude that they do not overwhelm the effect. The asymmetry $H_{2}^{n-p}$ is negative at large $x$. It reaches its maximum at $x \approx 0.8$ where it is $\sim 0.03 \mathrm{GeV}^{2}$, in agreement with the order of magnitude of the scale of $\mathrm{QCD}, \Lambda^{2}$. We also find that the $x$ dependence of $H_{2, \mathrm{~T}}^{n-p}$ is in poor agreement with the predictions of the IRR model. For more conclusive comparisons more precise data at $x \sim 0.4$ and $Q^{2} \sim 1 \mathrm{GeV}^{2}$ are necessary.

\section{Acknowledgments}

We are indebted to S. Brodsky, E. Gardi, A. Vainshtein, for discussions. We also thank A. Kaidalov and A. Szczurek for comunications. Two of us (S.A. and S.K.) are grateful to the University of Virginia, where the final part of this work was done, for hospitality. This work was supported by grants from the National Research Council COBASE program, 
from the U.S. Department of Energy under grant no. DE-FG02-01ER41200, and from the Russian Foundation for Basic Research under grant no. 03-02-17177.

[1] Proceedings of "The First International Workshop on Neutrino-Nucleus Interactions in the Few GeV Region", edited by J.G. Morfin, M. Sakuda and Y. Suzuki, to appear in Nucl. Phys. B, Proc. Suppl., September 2002.

[2] (a) S. I. Alekhin, Phys.Rev. D63 (2001) 094022; ibid, arXiv:hep-ph/0211096; ibid, JHEP 0302, 015 (2003) arXiv:hep-ph/0211294; (b) S. I. Alekhin, arXiv:hep-ph/0212370

[3] S. Liuti, R. Ent, C.E. Keppel and I. Niculescu, Phys.Rev.Lett. 89 (2002) 162001.

[4] Jefferson Laboratory Hall-Specific Conceptual Design Reports, http://www.jlab.org/div_dept/physics_division/pCDR_public

[5] K. G. Wilson, Phys. Rev. 179, 1499 (1969); E. V. Shuryak and A. I. Vainshtein, Nucl. Phys. B 199, 451 (1982).

[6] S. Alekhin, S. Kulagin and S. Liuti, in preparation.

[7] U.K. Yang and A. Bodek, Phys. Rev. Lett, 82 (1999) 2467; ibid Eur.Phys.J. C13 (2000) 241.

[8] L. L. Frankfurt and M. I. Strikman, Phys. Rep. 160, 236 (1988).

[9] M. Beneke, Phys. Rept. 317, 1 (1999) arXiv:hep-ph/9807443; M. Beneke and V.M. Braun, published in the Boris Ioffe Festschrift, "At the Frontier of Particle Physics/Handbook of QCD", M. Shifman editor, World Scientific (2001); arXiv:hep-ph/0010208.

[10] Y. L. Dokshitzer, G. Marchesini and B. R. Webber, Nucl. Phys. B 469, 93 (1996) arXiv:hep-ph/9512336;

E. Stein, M. Meyer-Hermann, L. Mankiewicz and A. Schafer, Phys. Lett. B 376, 177 (1996) arXiv:hep-ph/9601356;

M. Dasgupta and B. R. Webber, Phys. Lett. B 382, 273 (1996) arXiv:hep-ph/9604388.

[11] E. Stein, M. Maul, L. Mankiewicz and A. Schafer, Nucl. Phys. B 536, 318 (1998) arXiv:hep-ph/9803342.

[12] A.Szczurek and V.Uleshchenko, Eur.Phys.J. C12 (2000) 663.

[13] A. Capella, A. Kaidalov, C. Merino, J. Tran Thanh Van, Phys.Lett. B337 (1994) 358.

[14] S.J. Brodsky, hep-ph/0009228 hep-ph/0006310.

[15] H. Georgi and H. D. Politzer, Phys. Rev. D14, 1829 (1976).

[16] L. F. Abbott and R. M. Barnett, Annals Phys. 125, 276 (1980); L.F. Abbott, W.B. Atwood and R. M. Barnett, Phys. Rev. D22 (1980) 582; R. M. Barnett, Phys. Rev. Lett. 48, 1657 (1982).

[17] L.V. Gribov and Lipatov, Yad.Fiz. 20 (1975) 181; G. Altarelli and G. Parisi, Nucl.Phys. B126 (1977) 298; Dokshitzer, Sov.Phys.JETP 46 (1977) 641.

[18] D. I. Kazakov and A. V. Kotikov, Phys. Lett. B291 (1992) 171; W. L. van Neerven and E. B. Zilstra, Phys. Lett. B272 (1991) 127; Nucl. Phys. B383 (1992) 525.

[19] W. L. van Neerven and A. Vogt, Nucl. Phys. B568 (2000) 263; Phys. Lett. B490 (2000) 111.

[20] S. A. Larin, T. van Ritbergen and J. A. Vermaseren, Nucl. Phys. B 427 (1994) 41; S. A. Larin, P. Nogueira, T. van Ritbergen and J. A. Vermaseren, Nucl. Phys. B 492 (1997) 338, arXiv:hep-ph/9605317; A. Retey and J. A. Vermaseren, Nucl. Phys. B 604 (2001) 281, arXiv:hep-ph/0007294.

[21] S. Schaefer, A. Schafer and M. Stratmann, Phys. Lett. B 514 (2001) 284 arXiv:hep-ph/0105174; E. Gardi and R.G. Roberts, Nucl.Phys.B653 (2003) 227. 
[22] O. Nachtmann, Nucl. Phys. B63 (1973) 237.

[23] W. R. Frazer and J. F. Gunion, Phys. Rev. Lett. 45 (1980) 1138.

[24] A. De Rujula, H. Georgi and H.D. Politzer, Ann. Phys,. 103 (1977) 315.

[25] R.K. Ellis, W. Furmanski and R. Petronzio, Nucl.Phys.B212 (1983) 29; ibid Nucl.Phys. B207 (1982) 1; R.L. Jaffe and M. Soldate, Phys.Rev. D26 (1982) 49.

[26] S.Choi et al., Phys.Lett.B312 (1993) 351.

[27] A. L. Kataev, G. Parente and A. V. Sidorov, Nucl. Phys. B 573 (2000) 405 arXiv:hep-ph/9905310.

[28] G.B. West, Ann. Phys. 74 (1972) 464.

[29] S. V. Akulinichev, S. A. Kulagin, and G. M. Vagradov, Phys. Lett. B 158 (1985) 485; S. V. Akulinichev, S. Shlomo, S. A. Kulagin, and G. M. Vagradov, Phys. Rev. Lett. 55 (1985) 2239.

[30] S. A. Kulagin, Nucl. Phys. A 500 (1989) 653.

[31] C. Ciofi degli Atti and S. Liuti, Phys. Rev. C41 (1990) 1100.

[32] F. Gross and S. Liuti, Phys. Rev. C45 (1992) 1374.

[33] S.A. Kulagin, G. Piller and W. Weise, Phys. Rev. C50 (1994) 1154.

[34] S.A. Kulagin, Nucl. Phys. A 640 (1998) 435.

[35] W. Melnitchouk, A. W. Schreiber, and A. W. Thomas, Phys. Rev. D 49, 1183 (1994).

[36] J. Gomez, et al., Phys. Rev. D49 (1994) 4348.

[37] W. Melnitchouk, I. R. Afnan, F. Bissey, and A. W. Thomas, Phys.Rev.Lett. 84 (2000) 5455.

[38] L. W. Whitlow, E. M. Riordan, S. Dasu, S. Rock and A. Bodek, Phys. Lett. B282 (1992) 475 ;

A. C. Benvenuti et al. [BCDMS Collaboration], Phys. Lett. B223 (1989) 485;

A. C. Benvenuti et al. [BCDMS Collaboration], Phys. Lett. B237 (1990) 592;

M. Arneodo et al. [New Muon Collaboration], Nucl. Phys. B483 (1997) 3 hep-ph/9610231;

C. Adloff et al. [H1 Collaboration], Eur. Phys. J. C 21 (2001) 33 arXiv:hep-ex/0012053;

S. Chekanov et al. [ZEUS Collaboration], Eur. Phys. J. C 21 (2001) 443 arXiv:hep-ex/0105090.

[39] W. Giele et al., arXiv:hep-ph/0204316

[40] M.K. Gaidarov, M.V. Ivanov, A.N. Antonov, nucl-th/0207081.

[41] R. Machleidt, Phys.Rept. 149 (1987) 1.

[42] M. Lacombe et al., Phys.Rev. C21 (1980) 861.

[43] C. Ciofi degli Atti, E. Pace and G. Salmé, Phys.Rev. C43 (1991) 1155.

[44] X. D. Ji and P. Unrau, Phys. Rev. D 52 (1995) 72.

[45] NMC Collaboration, Nucl. Phys. B371 (1992) 3.

[46] L. W. Whitlow, S. Rock, A. Bodek, E. M. Riordan and S. Dasu, Phys. Lett. B 250, 193 (1990).

[47] M. Arneodo et al. [New Muon Collaboration], Nucl. Phys. B 487, 3 (1997) arXiv:hep-ex/9611022.

[48] E. D. Bloom and F. J. Gilman, Phys. Rev. Lett. 25, 1140 (1970);

E. D. Bloom and F. J. Gilman, Phys. Rev. D 4, 2901 (1971).

[49] The tables of the values of $H_{2, \mathrm{~T}}^{n-p}$ with the errors can be found in http://sirius.ihep.su/ alekhin/htasym/.

[50] We keep the lepton mass in Eq. (44) for the sake of completeness. Although this term is negligible in electron scattering, we take it into account in our analysis of muon data 
TABLE I: List of re-normalized experiments with the corresponding re-normalization factors $\eta$.

\begin{tabular}{ccc}
\hline \hline Experiment & \multicolumn{2}{c}{$\eta[\%]$} \\
\cline { 2 - 3 } & proton & deuterium \\
SLAC-E-49A & $1.5 \pm 1.3$ & $-1.1 \pm 1.2$ \\
SLAC-E-49B & $2.8 \pm 1.3$ & $0.2 \pm 1.2$ \\
SLAC-E-87 & $2.9 \pm 1.2$ & $0.9 \pm 1.2$ \\
SLAC-E-89B & $1.4 \pm 1.2$ & $-1.1 \pm 1.2$ \\
SLAC-E-139 & & $0.6 \pm 1.3$ \\
NMC $(90 \mathrm{GeV})$ & $-0.8 \pm 1.4$ & $-2.3 \pm 1.3$ \\
$\mathrm{NMC}(120 \mathrm{GeV})$ & $0.8 \pm 1.3$ & $-1.4 \pm 1.3$ \\
$\mathrm{NMC}(200 \mathrm{GeV})$ & $2.4 \pm 1.3$ & $0.1 \pm 1.3$ \\
$\mathrm{NMC}(280 \mathrm{GeV})$ & $1.2 \pm 1.3$ & $-1.0 \pm 1.3$ \\
\hline \hline
\end{tabular}

TABLE II: The Mellin moments of $H_{2, \mathrm{~T}}^{n-p}$.

\begin{tabular}{ccc}
\hline \hline $\mathrm{N}$ & $M_{2}^{n-p}(N)$ & $M_{\mathrm{T}}^{n-p}(N)$ \\
2 & $-0.0058 \pm 0.0069$ & $-0.012 \pm 0.014$ \\
3 & $-0.0046 \pm 0.0024$ & $-0.0048 \pm 0.0052$ \\
4 & $-0.0041 \pm 0.0013$ & $-0.0020 \pm 0.0026$ \\
5 & $-0.00355 \pm 0.00084$ & $-0.0008 \pm 0.0015$ \\
6 & $-0.00301 \pm 0.00061$ & $-0.00019 \pm 0.00096$ \\
7 & $-0.00254 \pm 0.00048$ & $0.00009 \pm 0.00068$ \\
8 & $-0.00215 \pm 0.00040$ & $0.00023 \pm 0.00052$ \\
9 & $-0.00183 \pm 0.00034$ & $0.00029 \pm 0.00041$ \\
10 & $-0.00157 \pm 0.00030$ & $0.00030 \pm 0.00034$ \\
\hline \hline
\end{tabular}




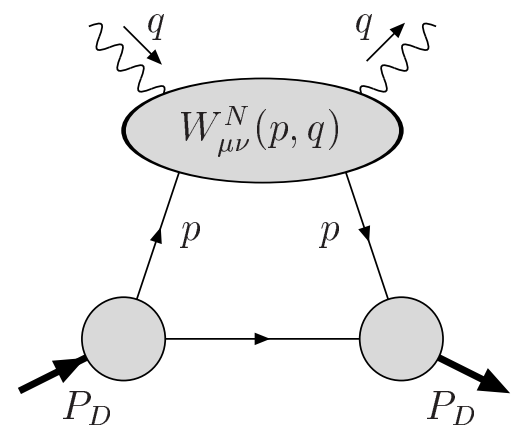

FIG. 1: Deuteron Compton scattering amplitude in incoherent scattering approximation. 

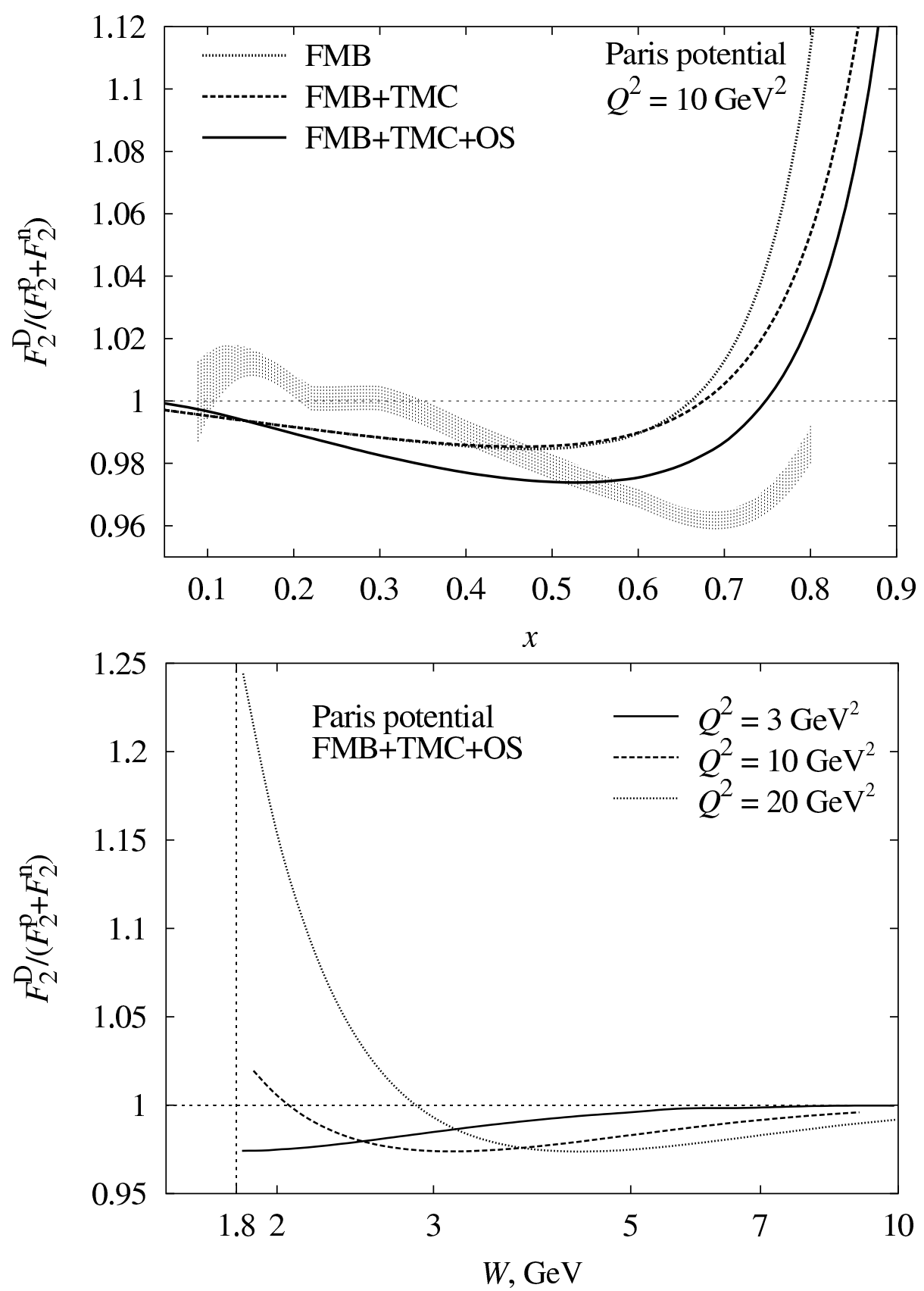

FIG. 2: The ratio $R_{2}^{D}$ calculated in different approximations. In the upper panel this ratio is presented as a function of $x$ for fixed $Q^{2}$ : Fermi motion and binding effects (dotted line), Fermi motion and binding effects combined with target mass corrections (dashed line); the full calculation including Fermi motion, binding, target mass and off-shell corrections is given by the solid line. The shaded area in the upper panel corresponds to the prediction of the nuclear density model of Ref. [36]. In the lower panel the ratio $R_{2}^{D}$ is shown as a function of $W$ for a few different $Q^{2}$. 


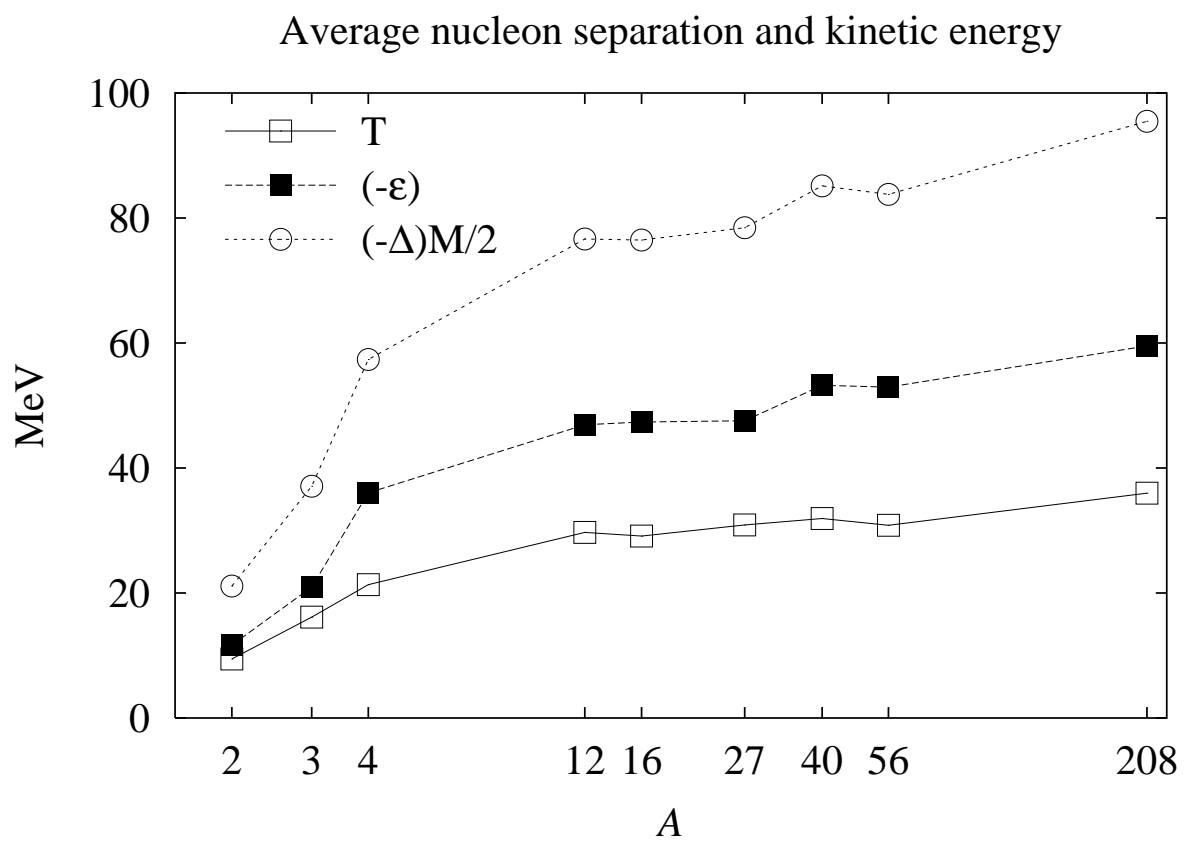

FIG. 3: Average nucleon kinetic and separation energy and off-shellness $\Delta$ as functions of the nuclear mass number $A$. 


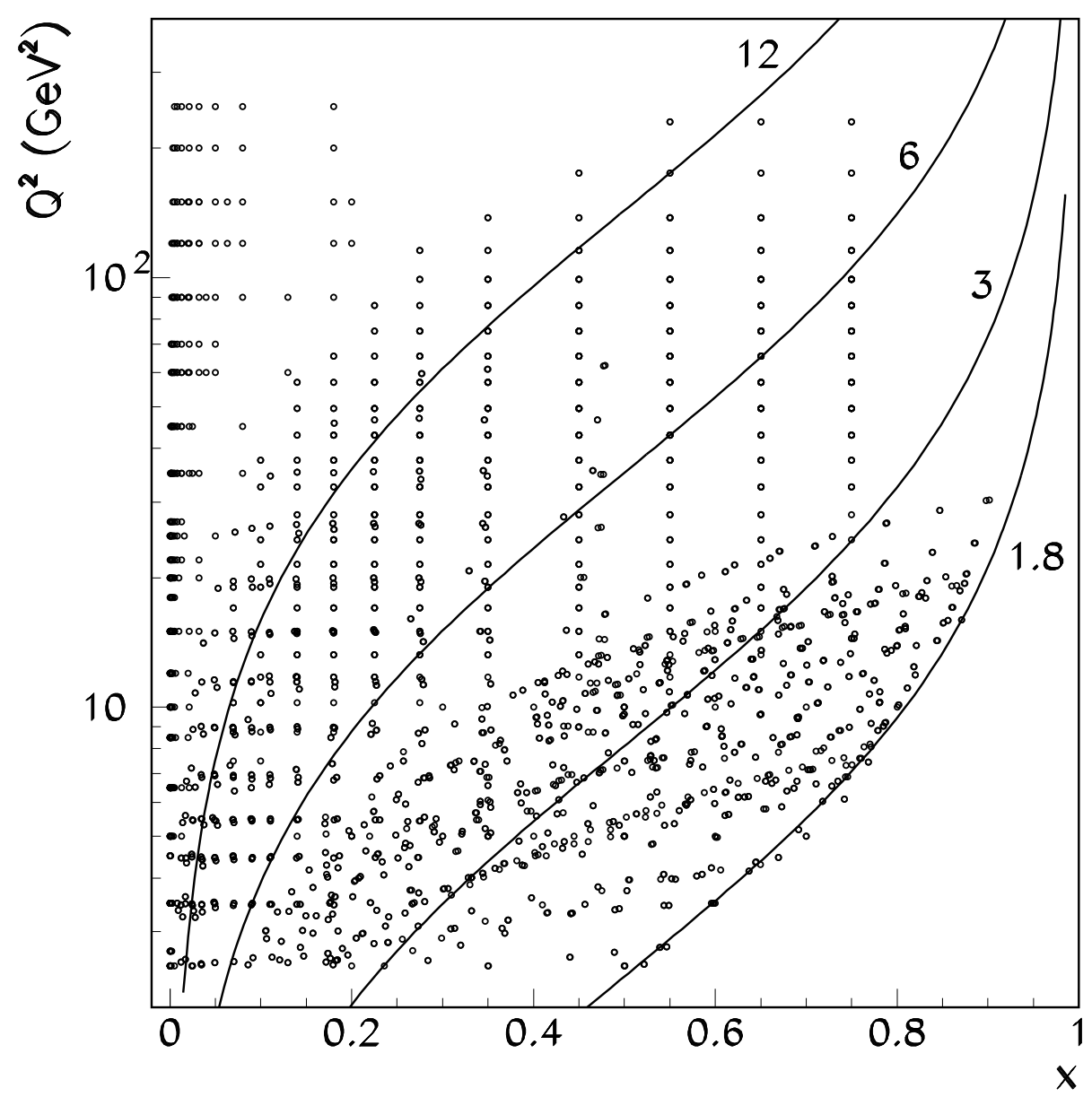

FIG. 4: Kinematic region of the data used in analysis. The curves correspond to constant values of the invariant mass $W$ whose values in units of $\mathrm{GeV}$ are indicated in the plot. 


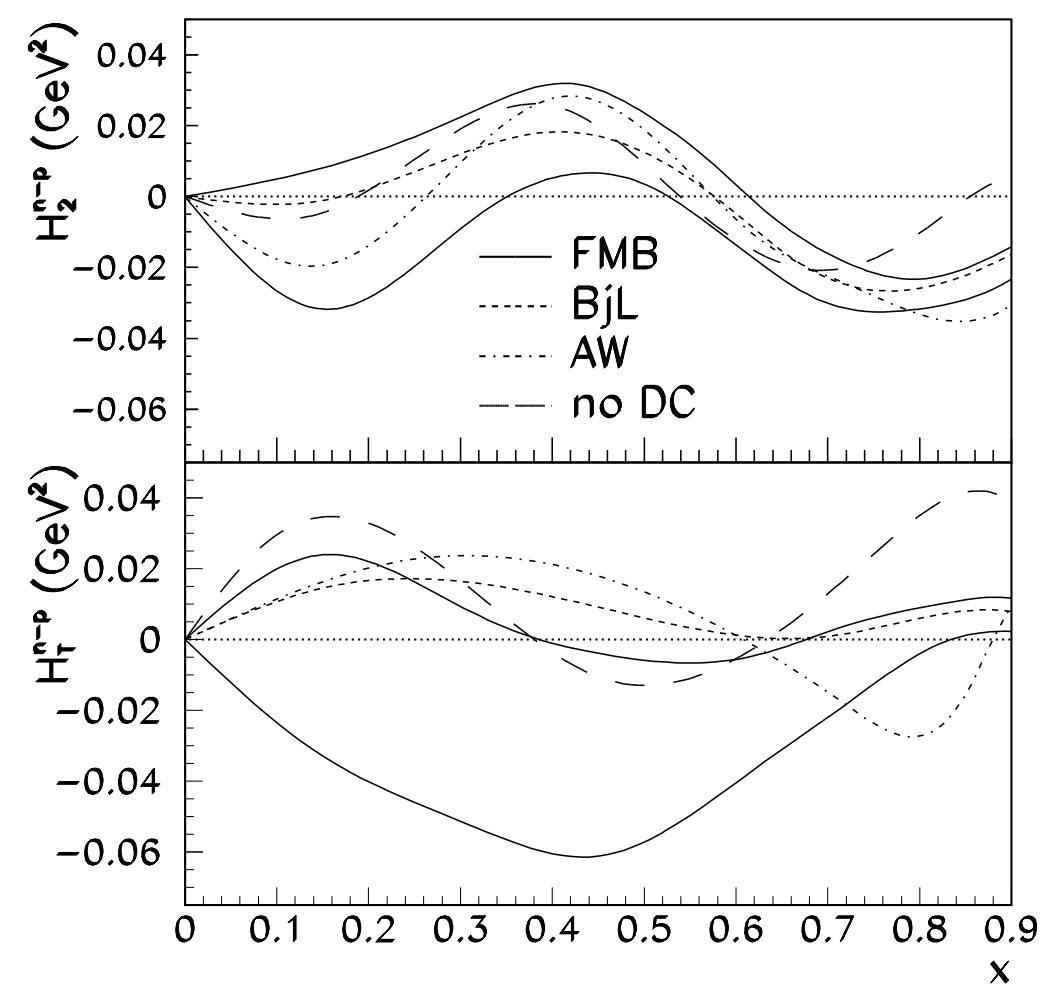

FIG. 5: Isospin asymmetries of the HT terms obtained using different treatments of Fermi motion and binding corrections: Eqs.(9) (delimited by solid lines, see text); Atwood-West [28] (dashdotted line); Eqs.(9) in the Bjorken limit, i.e. if all $1 / Q^{2}$ terms were disregarded (short dashes). The curve with long dashes shows the result without Fermi motion and binding corrections. 


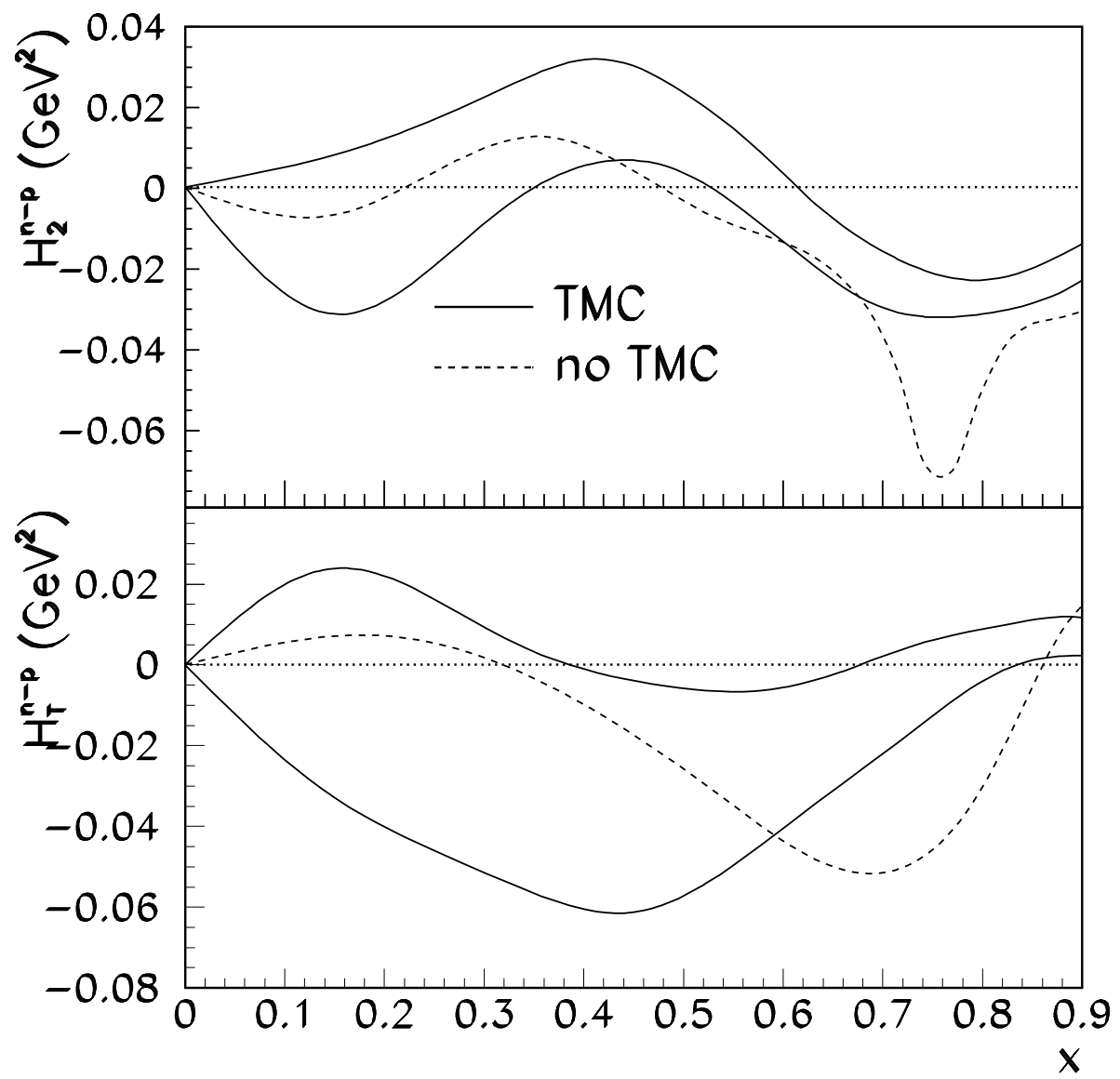

FIG. 6: Sensitivity of the isospin asymmetries to different approximations used in the calculation of the deuteron correction. Dashed line: fit with no TMC in the calculation of the deuteron correction; Area between solid lines: fit with full treatment of the TMC). 


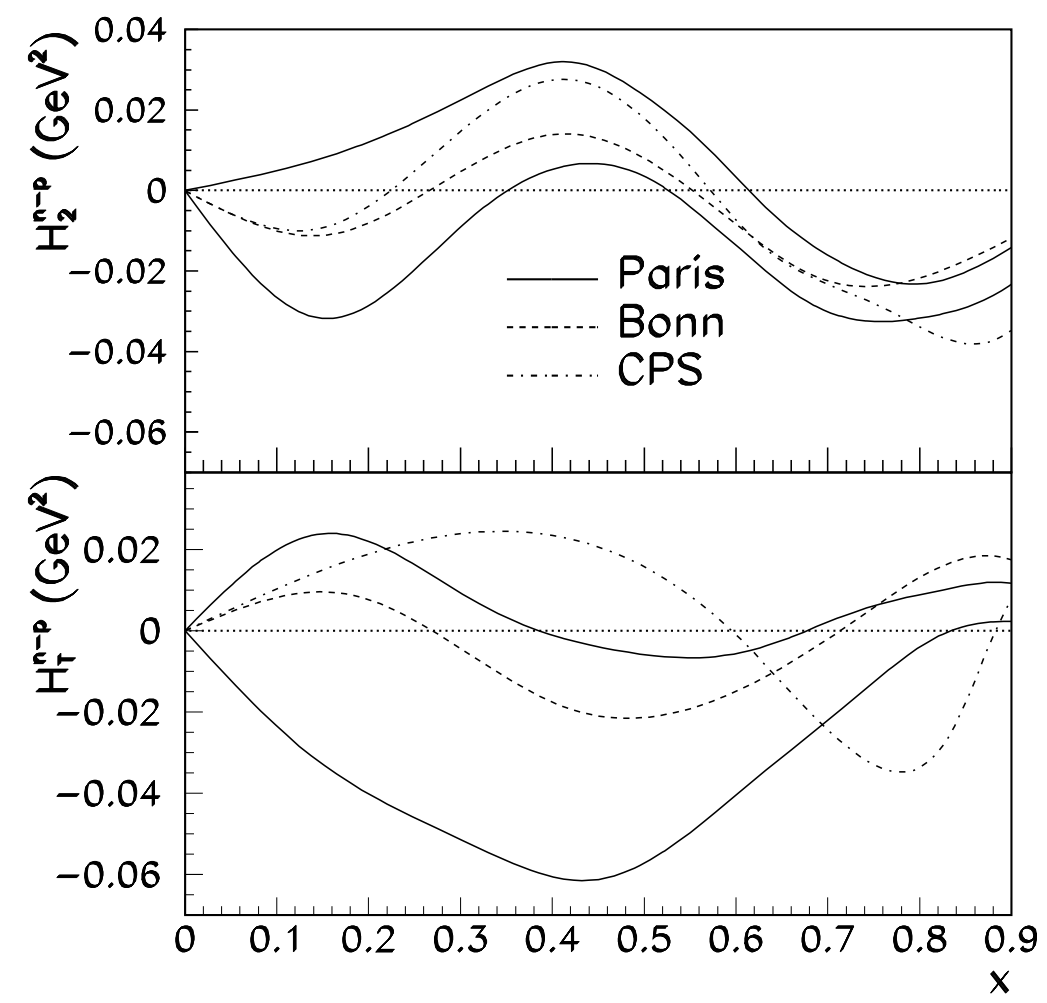

FIG. 7: Results of the fits with different deuteron wave functions: Ref. 42] (area between solid lines); Ref. 41] (dashed line); Ref. [43] (dashed-dotted line). 


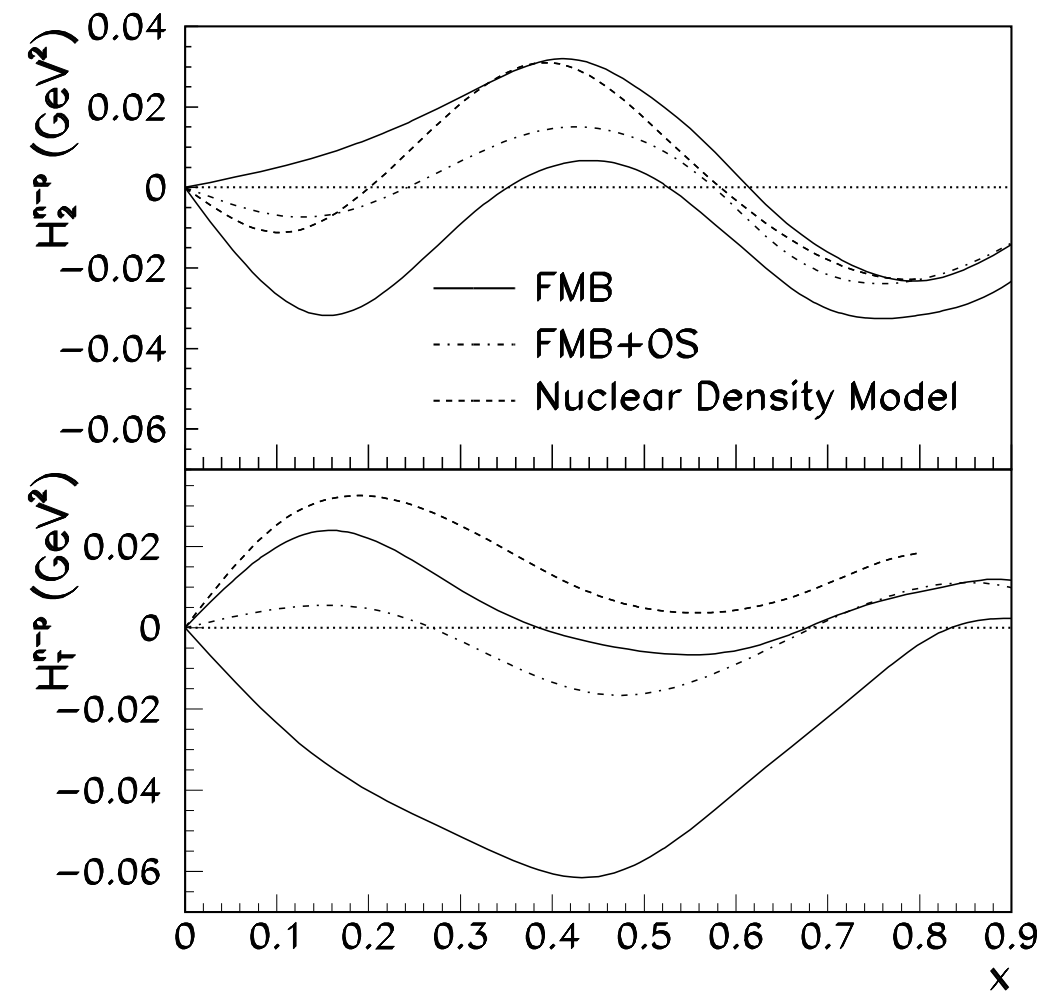

FIG. 8: Isospin asymmetries in the HT terms obtained within the incoherent scattering approximation (area between solid lines) as compared to the results obtained with the treatment of off-shell corrections (dashed-dotted lines). Results of the analysis based on the nuclear density model of Ref. [36] are given by the dashed lines. 


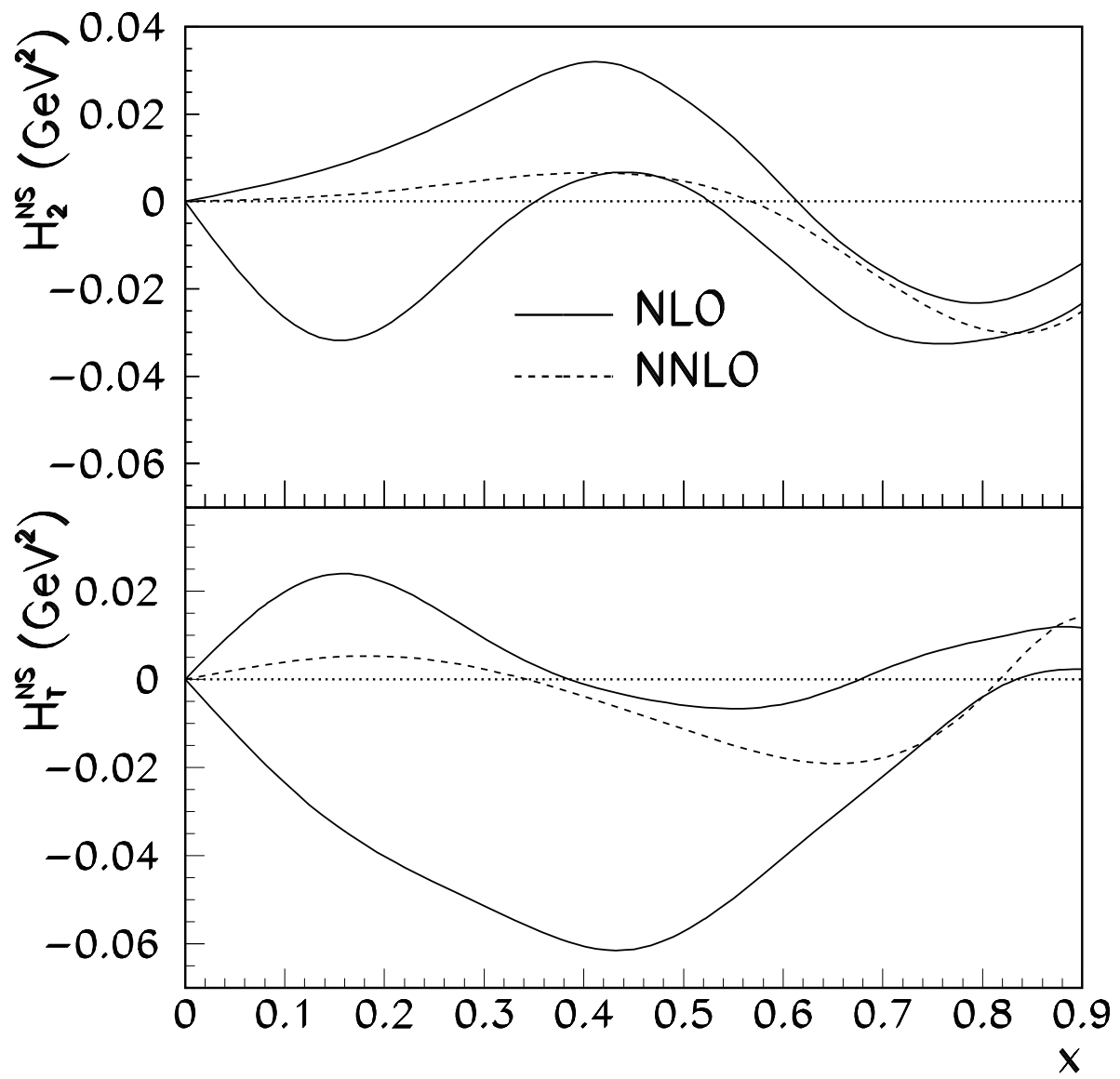

FIG. 9: Impact of NNLO QCD corrections on the isospin asymmetries of the HT terms: NLO QCD fit (area between solid lines); NNLO QCD fit (dashed lines). 


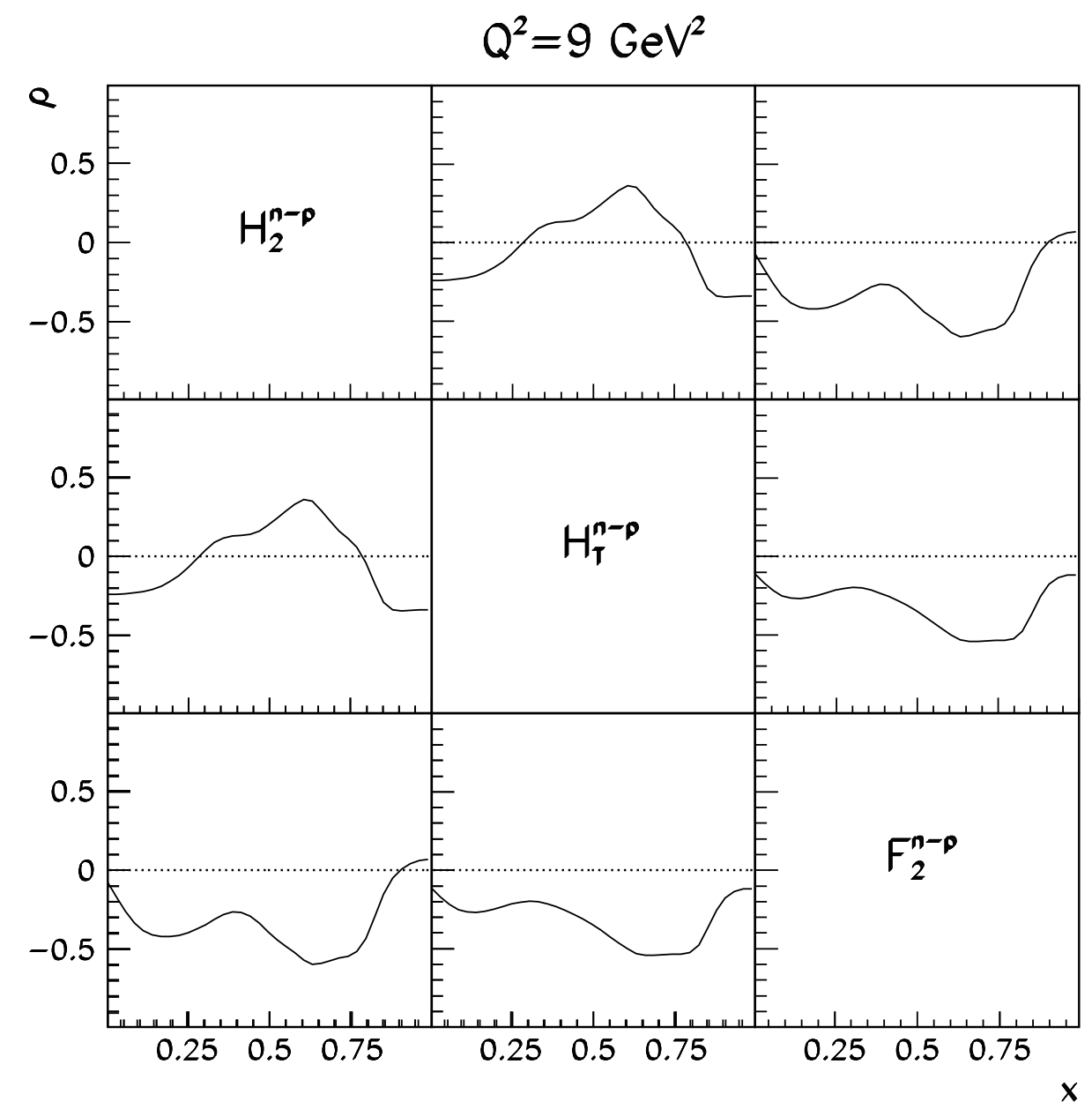

FIG. 10: The statistical correlation coefficients $\rho$ for the HT and LT terms determined from our fit. 


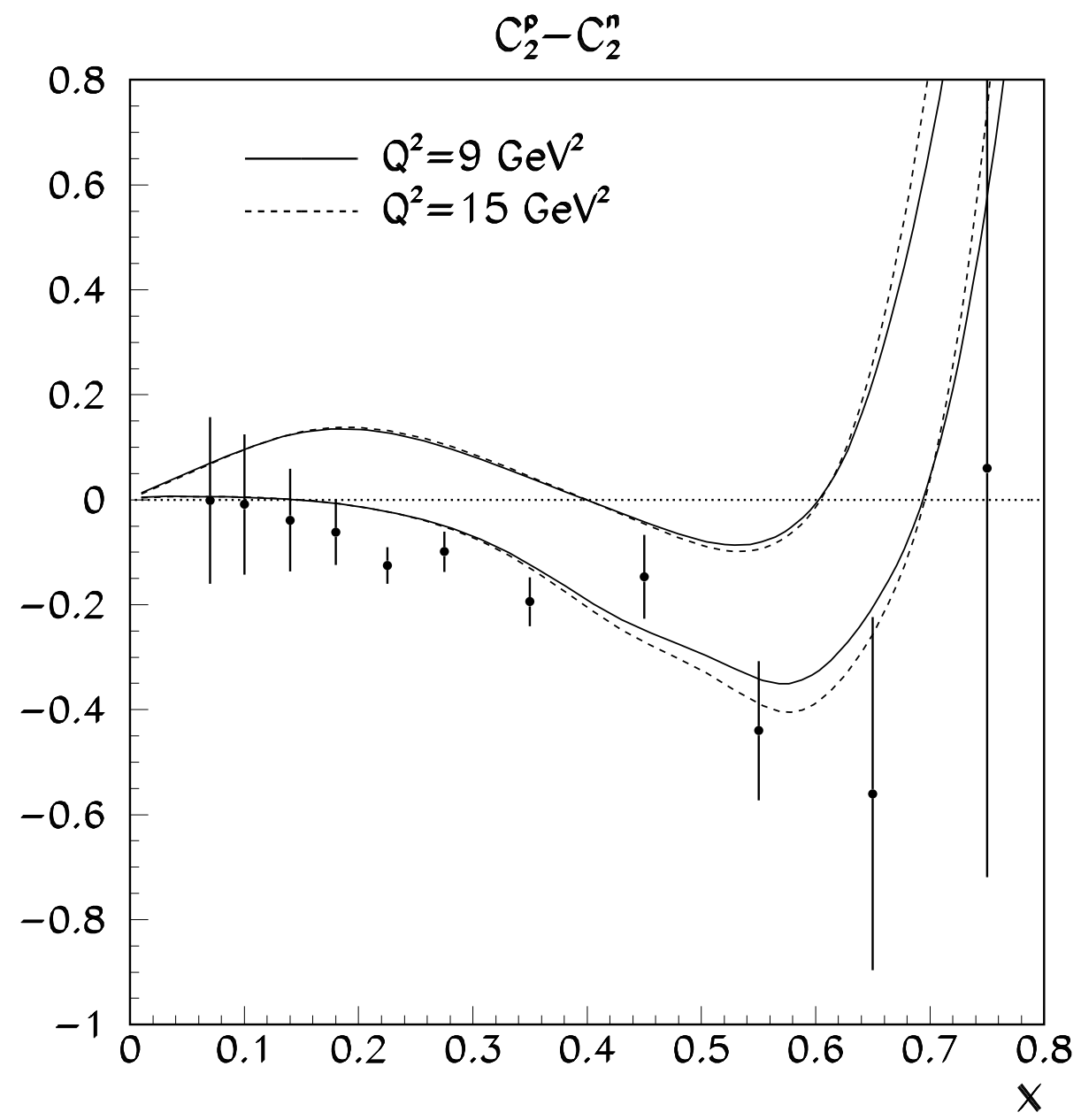

FIG. 11: The isospin asymmetry of the HT coefficients determined by NMC (points with errors bars) compared to to our determination (area inside error bands). 


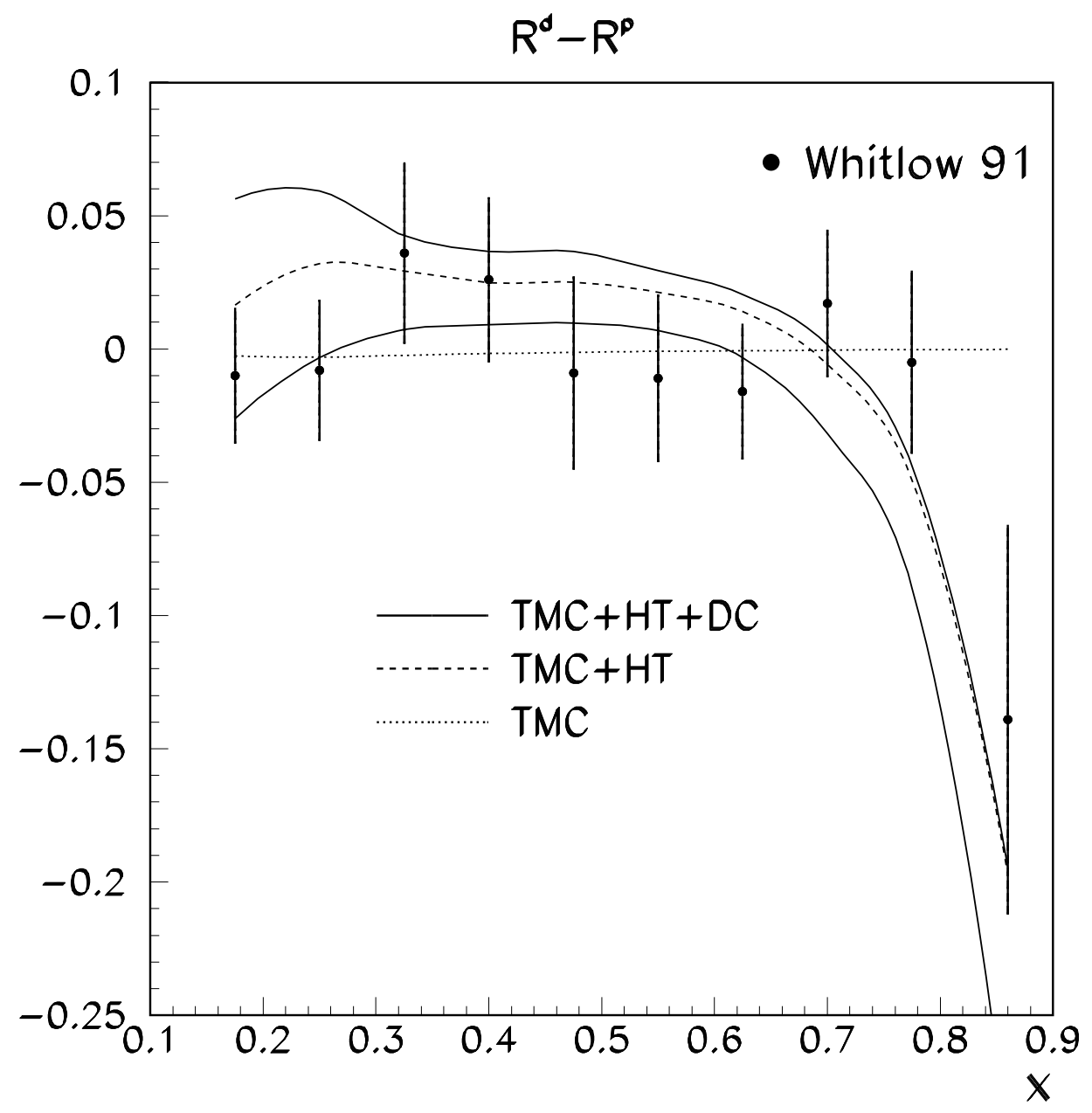

FIG. 12: The isospin asymmetry in the structure function $R$ determined from the SLAC data (points with error bars) compared to to our results. The LT contribution corrected for the TMC with no deuteron correction (dotted line); both the LT and HT terms but no deuteron corrections (dashed line); the same as above with the deuteron correction (area between solid lines). 


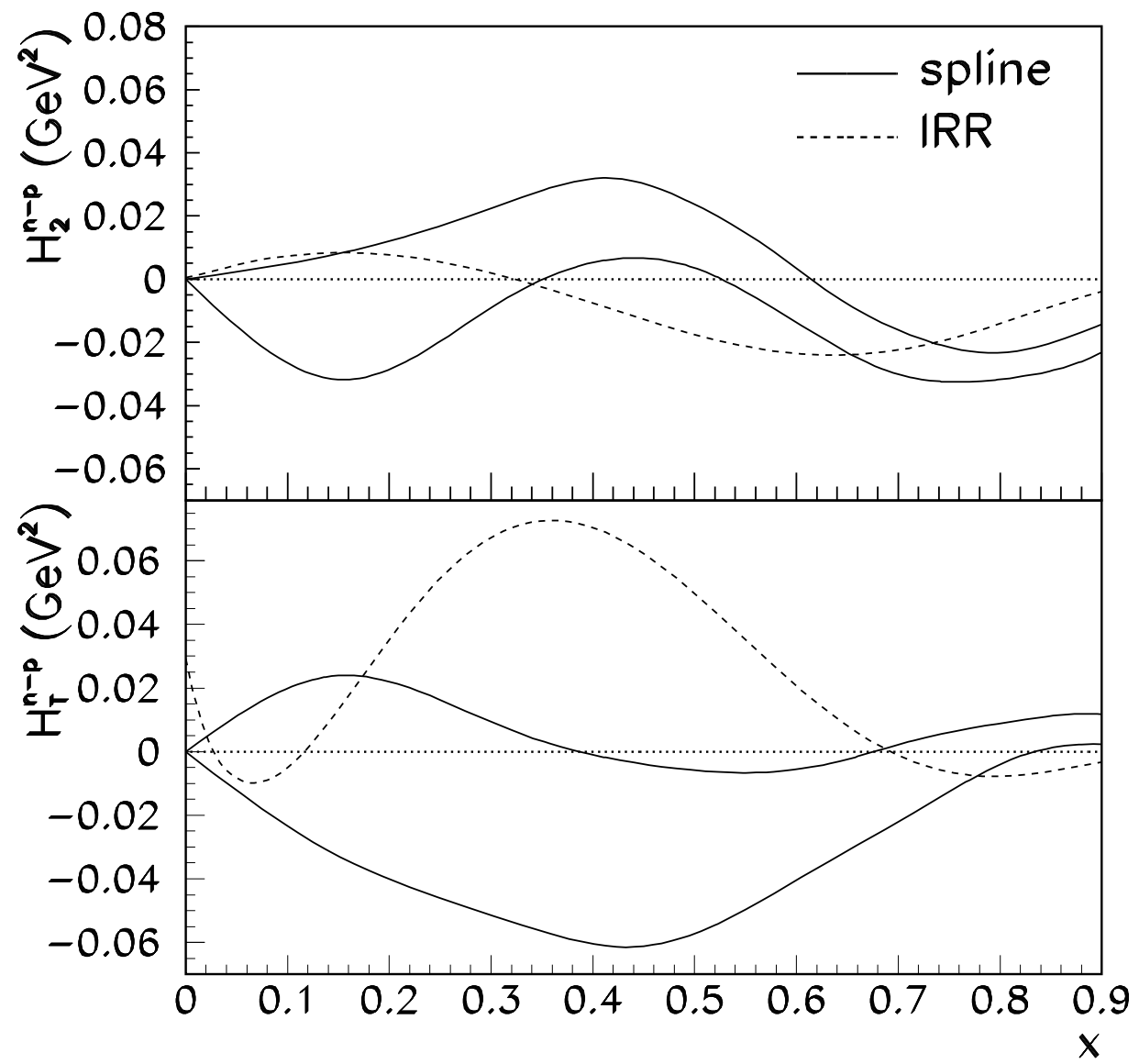

FIG. 13: Comparison of the phenomenological isospin asymmetries (area between solid lines) to the prediction of the infrared renormalon model (dashed lines). 


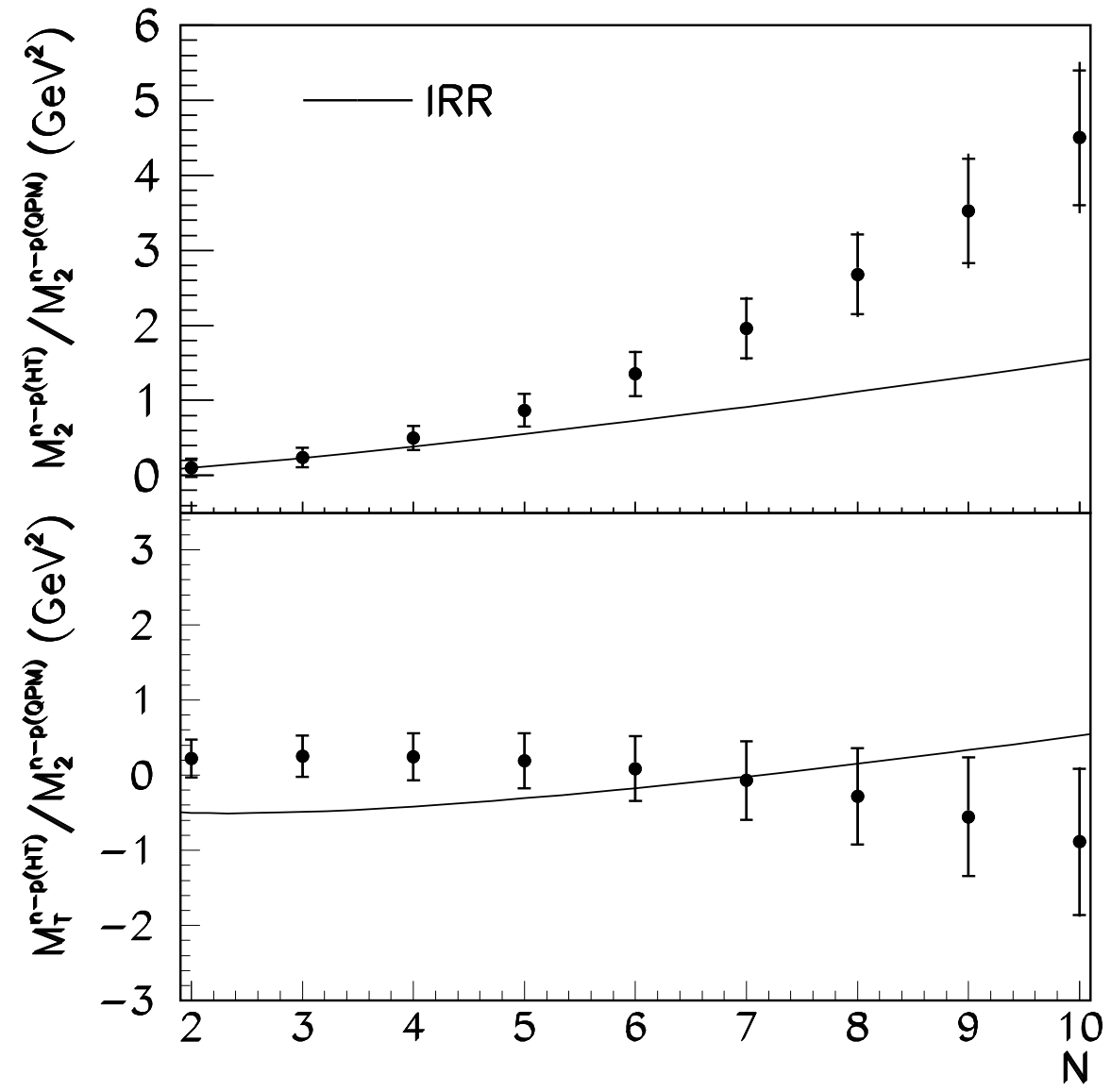

FIG. 14: The ratios of the Mellin moments of HT terms to the ones of LT terms (points with error bars) are compared with predictions of the IRR model (area between solid lines). The inner bars give total experimental errors in the moments, the outer bars include the error due to the extrapolation into the unmeasured region at $x>0.9$. 


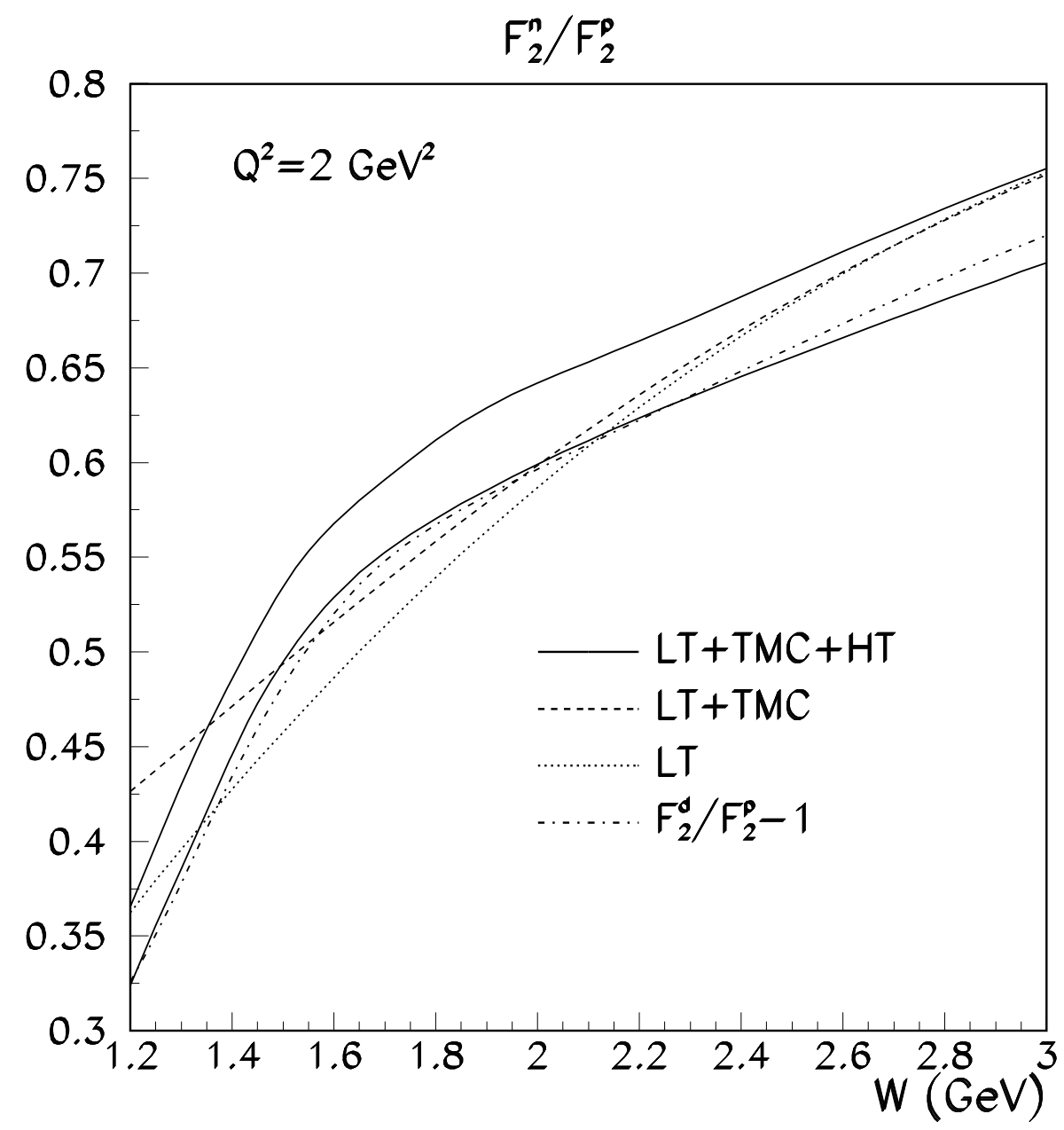

FIG. 15: The ratio $F_{2}^{n} / F_{2}^{p}$ calculated in different approximations and extrapolated to the resonance region: LT terms only (dotted line); effect of TMC (dashed line); TMC and HT terms (area between solid lines). 


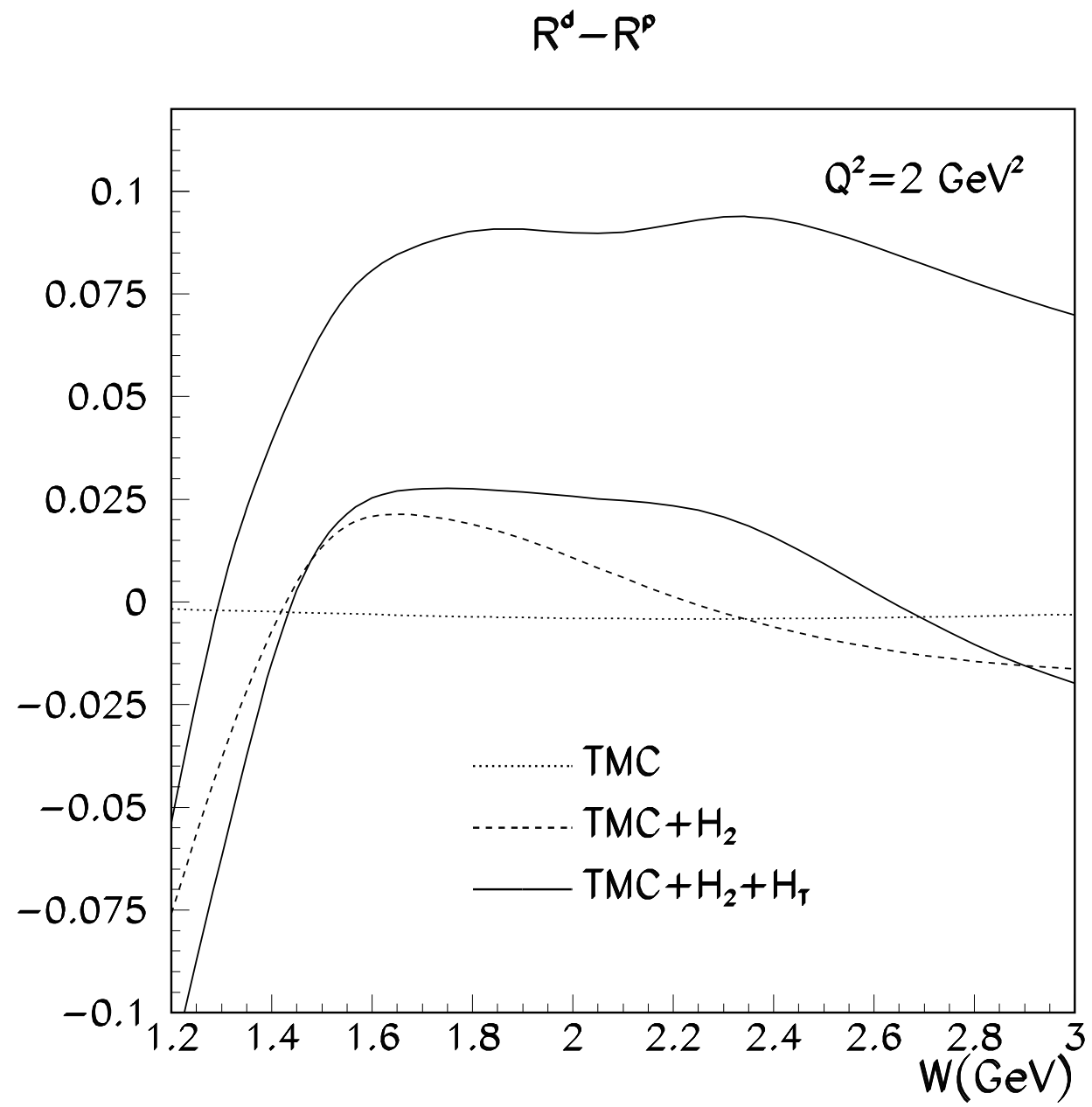

FIG. 16: The difference $R^{D}-R^{p}$ calculated in different approximations and extrapolated to the resonance region: only the LT terms with the TMC (dotted line); the same as above and the contribution from $H_{2}$ (dashed line); the same as the dashed line and the contribution from $H_{\mathrm{T}}$ (area between solid lines). 